\title{
Modelling the Effects of Competing Toxin Producing Phytoplankton on a Zooplankton Population: Role of Holling Type-II Functional Response with Time-Lag
}

\author{
O. P. Misra ${ }^{1}$, Chhatrapal Singh Sikarwar ${ }^{1, *}$, Poonam Sinha ${ }^{2}$
}

\author{
${ }^{1}$ School of Mathematics and Allied Sciences, Jiwaji University, Gwalior, 474 011, India \\ ${ }^{2}$ Department of Mathematics, S.M.S.Govt Model Science College, Gwalior, 474 011, India
}

\begin{abstract}
In this paper, a system consisting of two competing harmful phytoplankton and a zooplankton with Holling type-II functional response and discrete time lag is considered. A stable co-existence of all the species has been observed for the system without delay and the Hopf-bifurcation phenomenon is observed for the interior equilibrium point. The Hopf-bifurcating solution is obtained for the critical values of para meters like predation rates and half saturation constants. Further, using the normal form theory, we have determined the direction and the stability of the Hopf-bifurcation solution. The introduction of time delay in the system also shows the Hopf-bifurcation as the delay parameter passes through a critical value. Finally, the numerical simu lation is carried out to support the theoretical results.
\end{abstract}

Keywords Harmful Phytoplankton, Zooplankton, Delay, Stability, Hopf-Bifurcation

\section{Introduction}

There has been a global increase in harmful plankton blooms in last three decades[1-3]. Recently, there has been increasing interest in investigating the dynamics of harmful algal bloom (HAB) and its control[4 -7]. Plankton model is a kind of ecological model and different plankton models have been established and studied, previously[8, 9]. There are severalpapers in which attempts have been made to establish the role of different hydrological parameters in the formation of plankton blooms and then look for a suitable form of functional response to describe the reduction of zooplankton population due to toxin producing phytoplankton (TPP), for example, see[1, 2, 10, 11]. Reduction of grazing pressure of zooplankton due to release of toxic substances by phytoplankton is one of the most vital parameter in this context[12, 13]. Areas rich in some phytoplankton organisms e.g., Phaeocystis, Coscinodiscus, Rhizosolenia etc. are unaccepted/avoided by zooplankton due to dense concentration of phytoplankton or the production of some toxic as well as unpleasant factors by them and this phenomena is well explained by the exclusion principle[14, $15]$.

Buskey and Stockwell[16] have demonstrated in their field studies that micro and meso zooplankton population are

* Corresponding author:

chhatrapa184@gmail.com(Chhatrapal Singh Sikarwar)

Published online at http:/journal.sapub.org/ajcam

Copyright (C) 2012 Scientific \& Academic Publishing. All Rights Reserved reduced during the bloom of a chrysophyte Aureococcus nophagefferens in the southern Texas coast. These observations indicate that the toxic substance as well as toxic phytoplankton plays an important role on the growth of the zooplankton and have a great impact on phytoplankton zooplankton interactions. However, all the above studies do not explain several key features, such as, allelopathic interactions on the co-existence and persistence of phytoplankton species and its immed iate effect on predators, control of recurring harmful algal bloom or oscillations and the effect of time lag required to release toxic substances.

In this stage, we would like to mention that various combinations of predational functional response and toxin liberation process give rise to several interesting dynamics of the system. In this study, we are mainly interested to present a mechanis $m$ for planktonic bloom in which the liberation of toxic substance or the effect of toxic phytoplankton is not an instantaneous process but is mediated by some time lag and can be helpful to reduce oscillations in the population and in turn, it is useful to maintain a stable co-existence of the species. The study of biological systems with time delays have been of considerable interest for a long time. There are also several reports that the zooplankton mortality due to the toxic phytoplankton bloom occurs after some time lapse (see, www.mote.org, www.mdsg.umd.edu). Our mathematical and field observations [17] a lso suggest that the abundance of Paracalanus (zooplankton) population reduces after some time lapse of the bloom of toxic phytoplankton Noctiluca scintillans and this allows us some considerable freedom for considering the delay factor in the model construction. 
Sarkar et al.[18] studied the role of two toxin producing plankton and their effect on phytoplankton zooplankton system. They considered the predational functional response as a linear and distribution of toxic substances as Holling type II functions. They suggested in this paper that the ro le of time lag and environmental fluctuations in the two harmful phytoplankton-zooplankton dynamics may give some interesting results and needs further investigation. Therefore, in this paper we have studied a mathematical model for a phytoplankton-zooplankton system considering both the Holling type II predational functional response and Holling type II functional form with time lag for the distribution of toxic substances.

\section{Model Formulation and Basic Assumptions}

The current study has been originated from the theoretical as well as experimental results on the interaction of harmful algal bloom and different types of phytoplankton zooplankton interactions[10, 16-20]. Motivated from the literature and the field observations $[18,20]$, a dynamical model consisting of two competing phytoplankton harmful to zooplankton has been proposed and the role of time lag in the underlying system is studied. Let $P_{1}(t)$ and $P_{2}(t)$ are the concentrations of the two kinds of harmful phytoplankton and $Z(t)$ is the concentration of zooplankton at time t. Let $r_{1}$ and $r_{2}$ are the growth rates of the harmful phytoplankton, respectively, and $k$ is the carrying capacity, which is assumed to be common for both the phytoplankton species. Let $\rho_{1}$ and $\rho_{2}$ are the rates of predation of both phytoplankton by zooplankton and $\gamma_{1}$ and $\gamma_{2}$ are the corresponding conversion rates of zooplankton, where the predator functional response is assumed to follow the Holling type-II functional response[21, 22], with $h_{1}$ and $h_{2}$ as half saturation constants. Let $d$ is the natural death rate of zooplankton; $\alpha_{1}$ and $\alpha_{2}$ are the inhibitory effects of the two competing harmful phytoplankton. $\theta_{1}\left(\gamma_{1}>\theta_{1}\right)$ and $\theta_{2}\left(\gamma_{2}>\theta_{2}\right)$ are the rates of toxin liberation by the harmful phytoplankton reducing the growth of zooplankton. The discrete time lag $\tau$ is assumed to follow[23] and it is taken in the term describing the mortality of zooplankton due to liberation of toxic substances by two harmful phytoplankton $P_{1}, P_{2}$. The mathematical model is thus given by the following system of eSquations:

$$
\begin{gathered}
\frac{d P_{1}}{d t}=r_{1} P_{1}\left(1-P_{1} / k\right)-\alpha_{1} P_{1} P_{2}-\frac{\rho_{1} P_{1} Z}{\left(h_{1}+P_{1}\right)}, \\
\frac{d P_{2}}{d t}=r_{2} P_{2}\left(1-P_{2} / k\right)-\alpha_{2} P_{1} P_{2}-\frac{\rho_{2} P_{2} Z}{\left(h_{2}+P_{2}\right)}, \\
\frac{d Z}{d t}=\left(\frac{\gamma_{1} P_{1}}{\left(h_{1}+P_{1}\right)}+\frac{\gamma_{2} P_{2}}{\left(h_{2}+P_{2}\right)}\right) Z-d Z-\frac{\theta_{1} P_{1}(t-\tau) Z(t-\tau)}{\left(h_{1}+P_{1}(t-\tau)\right)} \\
-\frac{\theta_{2} P_{2}(t-\tau) Z(t-\tau)}{\left(h_{2}+P_{2}(t-\tau)\right)}
\end{gathered}
$$

System of Equations (1)-(3) is supplemented with the following initial conditions: $P_{1}(0) \geq 0, \quad P_{2}(0) \geq 0, Z(0) \geq 0$.

\section{Model Without Delay}

$$
\begin{gathered}
\frac{d P_{1}}{d t}=r_{1} P_{1}\left(1-P_{1} / k\right)-\alpha_{1} P_{1} P_{2}-\frac{\rho_{1} P_{1} Z}{\left(h_{1}+P_{1}\right)}, \\
\frac{d P_{2}}{d t}=r_{2} P_{2}\left(1-P_{2} / k\right)-\alpha_{2} P_{1} P_{2}-\frac{\rho_{2} P_{2} Z}{\left(h_{2}+P_{2}\right)}, \\
\frac{d Z}{d t}=\left(\frac{\left(\gamma_{1}-\theta_{1}\right) P_{1}}{\left(h_{1}+P_{1}\right)}+\frac{\left(\gamma_{2}-\theta_{2}\right) P_{2}}{\left(h_{2}+P_{2}\right)}\right) Z-d Z
\end{gathered}
$$

\subsection{Equlibria of the System}

The equilibrium points of the system of Equations (4)-(6) are

1. The trivial equilibrium point: $E_{T} \equiv(0,0,0)$,

2. The axial equilibrium points: $E_{A_{1}} \equiv(0, k, 0)$ and $E_{A_{2}} \equiv(k, 0,0)$

3. The boundary equilibrium points:

$$
\begin{gathered}
E_{B_{1}} \equiv\left[\frac{r_{2} k\left(r_{1}-\alpha_{1} k\right)}{r_{1} r_{2}-\alpha_{1} \alpha_{2} k^{2}}, \frac{r_{1} k\left(r_{2}-\alpha_{2} k\right)}{r_{1} r_{2}-\alpha_{1} \alpha_{2} k^{2}}, 0\right] \\
E_{B_{2}} \equiv\left(P_{1}^{\prime}, 0, Z^{\prime}\right),
\end{gathered}
$$

Where

$$
\begin{gathered}
P_{1}^{\prime}=d h_{1} /\left(\left(\gamma_{1}-\theta_{1}\right)-d\right), Z^{\prime}=\left(r_{1}\left(1-P_{1}^{\prime} / k\right)\left(h_{1}+P_{1}^{\prime}\right)\right) / \rho_{1} \\
E_{B_{3}} \equiv\left(0, P_{2}^{\prime \prime}, Z^{\prime \prime}\right),
\end{gathered}
$$

Where

$$
\begin{gathered}
P_{2}^{\prime \prime}=d h_{2} /\left(\left(\gamma_{2}-\theta_{2}\right)-d\right), \\
Z^{\prime \prime}=\left(r_{2}\left(1-P_{2}^{\prime \prime} / k\right)\left(h_{2}+P_{2}^{\prime \prime}\right)\right) / \rho_{2} .
\end{gathered}
$$

Now, we observe that the boundary equilibrium point $E_{B_{1}}$ exists if the inhibitory effects of both the harmful phytoplankton are less than certain thresholds, that is, if $\alpha_{1}<$ $\mathrm{r}_{1} / \mathrm{k}, \alpha_{2}<\mathrm{r}_{2} / \mathrm{k}$. The equilibriumpoints $E_{B_{2}}$ and $E_{B_{3}}$ exist if $\mathrm{d}<\left(\gamma_{1}-\theta_{1}\right)$ and $\mathrm{d}<\left(\gamma_{2}-\theta_{2}\right)$.

4. The unique interior equilibrium point: $\mathrm{E}^{*} \equiv\left(P_{1}^{*}, P_{2}^{*}, Z^{*}\right)$, where

$$
\begin{gathered}
P_{2}^{*}=\frac{h_{2}\left(d h_{1}+P_{1}^{*}\left(d-\left(\gamma_{1}-\theta_{1}\right)\right)\right)}{-h_{1} D_{2}+P_{1}^{*}\left(-D_{2}+\gamma_{1}-\theta_{1}\right)}, \\
Z=\frac{\left(P_{1}^{*}+h_{1}\right)\left(P_{1}^{* 2} d_{1}+P_{1}^{*} d_{2}+d_{3}\right)}{-k h_{1} D_{2}+P_{1}^{*}\left(-D_{2}+\left(\gamma_{1}-\theta_{1}\right)\right)}, \\
d_{1}=r_{1}\left(D_{2}-\left(\gamma_{1}-\theta_{1}\right)\right), \\
d_{3}=-k h_{1}\left(d h_{2} \alpha_{1}+r_{1} D_{2}\right), \\
d_{2}=\left(k h_{2} \alpha_{1}\left(-d+\left(\gamma_{1}-\theta_{1}\right)\right)+r_{1}\left(h_{1} D_{2}+k\left(-D_{2}+\gamma_{1}-\theta_{1}\right)\right),\right. \\
\text { and } P_{1}^{*} \text { is given by } \\
\delta_{1} P_{1}^{* 3}+\delta_{2} P_{1}^{* 2}+\delta_{3} P_{1}^{*}+\delta_{4}=0, \delta_{1}=r_{1}\left(-D_{2}+\left(\gamma_{1}-\theta_{1}\right)^{2} \rho_{2}\right. \\
\delta_{2}=-\left(D_{2}-\left(\gamma_{1}-\theta_{1}\right)\right)\left(-r_{1}\left(2 h_{1} D_{2}+\left(\left(\gamma_{1}-\theta_{1}\right)\right)\right) \rho_{2}-k h_{2}\left(\alpha_{2}\left(\gamma_{2}-\theta_{2}\right) \rho_{1}\right.\right. \\
\left.\left.\left.\left.+\alpha_{1}\left(-d+\left(\gamma_{1}-\theta_{1}\right)\right) \rho_{2}\right)\right) \rho_{2}-k h_{2}\left(\alpha_{2}\left(\gamma_{2}-\theta_{2}\right) \rho_{1}+\alpha_{1}\left(\gamma_{1}-\theta_{1}\right)\right) \rho_{2}\right)\right) \\
\delta_{3}=h_{2}{ }^{2} r_{2}\left(-d+\left(\gamma_{1}-\theta_{1}\right)\right)\left(\gamma_{2}-\theta_{2}\right) \rho_{1}+h_{1} r_{1} D_{2}\left(h_{1} D_{2}+\right. \\
\left.\left(-D_{2}+\left(\gamma_{1}-\theta_{1}\right)\right)\right) \rho_{2}+k h_{2}\left(r_{2}\left(\gamma_{2}-\theta_{2}\right)\left(-D_{2}+\left(\gamma_{1}-\theta_{1}\right)\right) \rho_{1}\right. \\
\left.+h_{1}\left(\alpha_{2} D_{2}\left(\gamma_{2}-\theta_{2}\right) \rho_{1}+\alpha_{1}\left(\left(\gamma_{1}-\theta_{1}\right)\left(d+D_{2}\right)-2 d D_{2}\right) \rho_{2}\right)\right),
\end{gathered}
$$




$$
\begin{aligned}
& \delta_{4}=-h_{1}\left(d h_{2}{ }^{2} r_{2}\left(\gamma_{2}-\theta_{2}\right) \rho_{1}+k h_{1} r_{1} D_{2}^{2} \rho_{2}+\right. \\
& \left.k h_{2} D_{2}\left(r_{2}\left(\gamma_{2}-\theta_{2}\right) \rho_{1}+d h_{1} \alpha_{1} \rho_{2}\right)\right), D_{2}=\left(d-\left(\gamma_{2}-\theta_{2}\right)\right) .
\end{aligned}
$$

The unique interior equilibrium point $E^{*}$ exist if

(i) $d \geq\left(\gamma_{2}-\theta_{2}\right),\left(\gamma_{1}-\theta_{1}\right)<d,\left(\gamma_{1}-\theta_{1}\right)+\left(\gamma_{2}-\theta_{2}\right)>d$,

(ii) $k h_{2} \alpha_{1} \rho_{2}\left(d-\left(\gamma_{1}-\theta_{1}\right)\right)>r_{1}\left(2 h_{1} D_{2}\right.$

$$
\left.+k\left(-d+\left(\gamma_{1}-\theta_{1}\right)+\left(\gamma_{2}-\theta_{1}\right)+\left(\gamma_{2}-\theta_{2}\right)\right)\right) \rho_{2}
$$$$
+k h_{2} \alpha_{2} \rho_{1}\left(\gamma_{2}-\theta_{2}\right)\left(-d+\left(\gamma_{1}-\theta_{1}\right)+\left(\gamma_{2}-\theta_{2}\right)\right) \text {, }
$$

(iii) $h_{1} r_{1} D_{2}\left(h_{1} D_{2}+2 k\left(-d+\left(\gamma_{1}-\theta_{1}\right)+\left(\gamma_{2}-\theta_{2}\right)\right)\right) \rho_{2}$

$+k h_{2}\left(r_{2}\left(\gamma_{2}-\theta_{1}\right)\left(-d+\left(\gamma_{1}-\theta_{1}\right)+\left(\gamma_{2}-\theta_{2}\right)\right) \rho_{1}\right.$

$\left.+h_{1}\left(\alpha_{2} D_{2}\left(\gamma_{2}-\theta_{1}\right) \rho_{1}+\alpha_{1}\left(\left(\gamma_{1}-\theta_{1}\right)\left(2 d-\left(\gamma_{2}-\theta_{2}\right)\right)\right) \rho_{2}\right)\right)$

$>h_{2}^{2} r_{2}\left(d-\left(\gamma_{1}-\theta_{1}\right)\right)\left(\gamma_{2}-\theta_{2}\right) \rho_{1}+2 h_{1} k h_{2} \alpha_{1} \rho_{2} d D_{2}$

(iv) $P_{1}^{*}\left(r_{1}\left(h_{1} D_{2}+k\left(-d+\left(\gamma_{1}-\theta_{1}\right)+\left(\gamma_{2}-\theta_{2}\right)\right)\right)\right)$

$>P_{1}^{*} k h_{2} \alpha_{1}\left(-d+\left(\gamma_{1}-\theta_{1}\right)\right) k h_{1}\left(d h_{2} \alpha_{1}+r_{1} D_{2}\right)$

$+P_{1}^{* 2} r_{1}\left(-d+\left(\gamma_{1}-\theta_{1}\right)+\left(\gamma_{2}-\theta_{2}\right)\right)$.

\subsection{Dynamic Behaviour and Hopf-Bifurcation}

Eigen value analysis to establish local asymptotic stability: By computing the variational matrix around the respective biological feasible equilibria, one can easily deduce the following lemmas:

Lemma 3.2.1 The steady state $E_{T}(0,0,0)$ of system of Equations (4)-(6) is a saddle point.

Le mma 3.2.2 There exists two steady states $E_{A_{1}}(0, k, 0)$ and $E_{A_{2}}(\mathrm{k}, 0,0)$ which are feasible (one harmful phytoplankton and zooplankton free state) and are unstable saddle.

Lemma 3.2.3 There exists a zooplankton free steady state $E_{B_{1}}$ which is unstable saddle if $\alpha_{1} / \alpha_{2}<r_{1} / r_{2}$.

Lemma 3.2.4 There exists a steady state $E_{B_{2}}$ which is unstable if

$$
r_{2}-\alpha_{2} P_{1}^{\prime \prime}-\rho_{2} Z^{\prime \prime} / h_{1}>0 .
$$

Lemma 3.2.5 There exists a steady state $E_{B_{3}}$ which is unstable if

$$
r_{1}-\alpha_{1} P_{2}^{\prime \prime}-\frac{\rho_{2} Z^{\prime \prime}}{h}>0 .
$$

Next, we perform a stability study of the interior equilibrium point $E^{*}$. For the sake of simplicity, the equilibrium point $\left(P_{1}^{*}, P_{2}^{*}, Z^{*}\right)$ of the system of Equations (4)-(6) is shifted to a new point $\left(x_{1}, x_{2}, x_{3}\right)$ through transformations

$$
x_{1}=P_{1}-P_{1}^{*}, x_{2}=P_{2}-P_{2}^{*}, x_{3}=Z-Z^{*}
$$

In terms of the new variables, the dynamical equations can be written in the matrix form as

$$
\dot{X}=A X+B
$$

where $\operatorname{dot}($.$) over \mathrm{X}$ denotes the derivative with respect to time. Here $\mathrm{AX}$ is the linear part of the system and $\mathrm{B}$ represents the non linear part. Moreover,

$$
X=\left[\begin{array}{l}
x_{1} \\
x_{2} \\
x_{3}
\end{array}\right], \quad A=\left[\begin{array}{ccc}
-P_{1}^{*} a_{11} & -P_{1}^{*} \alpha_{1} & -P_{1}^{*} H_{1} \rho_{1} \\
-P_{2}^{*} \alpha_{2} & -P_{2}^{*} a_{22} & -P_{2}^{*} H_{2} \rho_{2} \\
c_{1} Z^{*} & c_{2} Z^{*} & 0
\end{array}\right]
$$

and

$$
B=\left[\begin{array}{c}
x_{1}\left(b_{1} x_{1}+b_{2} x_{2}+b_{3} x_{2}+b_{3} x_{3}+b_{4} x_{1}^{2}+b_{5} x_{1} x_{3}\right) \\
x_{2}\left(r_{1} x_{2}+n_{2} x_{1}+n_{3} x_{3}+n_{4} x_{2}^{2}+n_{5} x_{2} x_{3}\right) \\
x_{1} Q_{1}+x_{2}\left(q_{3} x_{2}+q_{6} x_{2}{ }^{2}+q_{7} x_{3}+q_{6} x_{2} x_{3}\right)
\end{array}\right]
$$

where,

$$
\begin{gathered}
Q_{1}=\left(q_{1} x_{1}+q_{2} x_{1}^{2}+q_{3} x_{1} x_{3}\right), a_{11}=r_{1} / k-Z^{*} H_{1}^{2} \rho_{1}, \\
c_{2}=h_{2} H_{2}^{2}\left(\gamma_{2}-\theta_{2}\right), \quad a_{22}=r_{2} / k-Z^{*} H_{2}^{2} \rho_{2}, \\
c_{1}=h_{1} H_{1}^{2}\left(\gamma_{1}-\theta_{1}\right), \\
c_{2}=h_{2} H_{2}^{2}\left(\gamma_{2}-\theta_{2}\right), b_{1}=\left(-r_{1} / k+Z^{*} h_{1} \rho_{1} H_{1}^{3}\right) \\
c_{2}=h_{2} H_{2}^{2}\left(\gamma_{2}-\theta_{2}\right), b_{1}=\left(-r_{1} / k+Z^{*} h_{1} \rho_{1} H_{1}^{3}\right) \\
b_{1}=\left(-r_{1} / k+Z^{*} h_{1} \rho_{1} H_{1}^{3}\right), \quad b_{2}=-\alpha_{1}, \quad b_{3}=-\rho_{1} h_{1} H_{1}^{2}, \\
b_{4}=-Z^{*} H_{1}^{4} \rho_{1} h_{1}, \quad b_{5}=\rho_{1} h_{1} H_{1}^{3}, \\
n_{1}=\left(\frac{-r_{2}}{k}+Z^{*} h_{2} \rho_{2} H_{2}^{3}\right), \quad n_{2}=-\alpha_{2}, \quad n_{3}=-\rho_{2} h_{2} H_{2}^{2}, \\
n_{4}=-Z^{*} H_{2}^{3} \rho_{2} h_{2}, \quad n_{5}=\rho_{2} h_{2} H_{2}^{3} \\
q_{1}=-c_{1} Z^{*} H_{1}, \quad q_{2}=c_{1} Z^{*} H_{1}^{2}, \quad q_{3}=c_{1}, q_{4}=-c_{1} H_{1}, \\
H_{1}=\frac{1}{\left(h_{1}+P_{1}^{*}\right)}, q_{5}=-c_{2} Z^{*} H_{2}, \\
q_{6}=c_{2} Z^{*} H_{2}^{2}, q_{7}=c_{2}, H_{2}=\frac{1}{\left(h_{2}+P_{2}^{*}\right)}, q_{8}=-c_{2} H_{2} .
\end{gathered}
$$

The characteristic equation of the community matrix corresponding to the linearized version of system of Equations (4)-(6) at $E^{*}$ is

$$
\lambda^{3}+A_{1} \lambda^{2}+A_{2} \lambda+A_{3}=0
$$

Where

$$
\begin{gathered}
A_{1}=a_{11} P_{1}^{*}+a_{22} P_{2}^{*}, \\
A_{2}=\left(a_{11} a_{22}-\alpha_{1} \alpha_{2}\right) P_{1}^{*} P_{2}^{*}+c_{2} \rho_{2} H_{2} P_{2}^{*} Z^{*}+c_{1} \rho_{1} H_{1} P_{1}^{*} Z^{*} \\
A_{3}=\left(a_{11} c_{2} \rho_{2} H_{2}+a_{22} c_{1} \rho_{1} H_{1}-c_{1} \rho_{2} H_{2} \alpha_{1}-c_{2} \rho_{1} H_{1} \alpha_{2}\right) P_{1}^{*} P_{2}^{*} Z^{*}
\end{gathered}
$$

Using the Routh-Hurwitz criteria[22, 24], $E^{*}$ is locally asymptotically stable, if $A_{1}>0, A_{3}>0$ and $A_{1} A_{2}>A_{3}$. Here the conditions $A_{1}>0, A_{3}>0$ and $\mathrm{A}_{1} \mathrm{~A}_{2}>A_{3}$ requires,

(i) $Z^{*} \leq \min \left(\frac{r_{1}}{\rho_{1} k H_{1}^{2}}, \frac{r_{2}}{\rho_{2} k H_{2}^{2}}\right)$

(ii) $\frac{\alpha_{1}}{a_{11}}<\frac{c_{2}}{c_{1}}<\frac{a_{22}}{\alpha_{2}}$.

If one of the above mentioned conditions is violated then the system would become unstable around the positive interior equilibrium $E^{*}$.

Now, we will study the Hopf-bifurcation[1 1, 25, 26] of the system of Equations (4)-(6) taking $\rho_{2}$ as the bifurcation 
parameter. The necessary and sufficient conditions for the existence of the Hopf-bifurcation for $\rho_{2}=\rho_{2}{ }^{*}$, if it exist are

(i) $A_{\mathrm{i}}\left(\rho_{2}{ }^{*}\right)>0, \mathrm{i}=1,2,3$ (ii) $A_{1}\left(\rho_{2}{ }^{*}\right) A_{2}\left(\rho_{2}{ }^{*}\right)-A_{3}\left(\rho_{2}{ }^{*}\right)=0$ and (iii) the eigenvalues of the characteristic equation should be of the form $\lambda_{\mathrm{i}}=\mathrm{u}_{\mathrm{i}}+i \mathrm{v}_{\mathrm{i}}$, and $\frac{d u}{d \rho_{2}} \neq 0, \mathrm{i}=1,2,3$. After substituting the values, the condition $\mathrm{C}=\mathrm{A}_{1} \mathrm{~A}_{2}-\mathrm{A}_{3}$ becomes

$$
=-B_{1} \rho_{2}^{2}+B_{2} \rho_{2}+B_{3}=0
$$

$$
\begin{gathered}
B_{1}=N_{2} N_{4}, \quad B_{2}=N_{1} N_{4}-N_{2} N_{3}-N_{6}, \quad B_{3}=N_{1} N_{3}-N_{5}, \\
N_{1}=a_{11} P_{1}^{*}+\frac{r_{2}}{k} P_{2}^{*}, \quad N_{2}=Z H_{2}^{2} P_{2}^{*}, \\
N_{3}=\left(a_{11} \frac{r_{2}}{k}-\alpha_{1} \alpha_{2}\right) P_{1}^{*} P_{2}^{*}+c_{1} \rho_{1} H_{1} P_{1}^{*} Z^{*}, \\
N_{4}=c_{2} H_{2} P_{2}^{*} Z^{*}-a_{11} P_{1}^{*} P_{2}^{*} Z^{*} H_{2}^{2}, \\
N_{5}=\left(\frac{r_{2}}{k} c_{1} \rho_{1} H_{1}-c_{2} \rho_{1} H_{1} \alpha_{2}\right) P_{1}^{*} P_{2}^{*} Z^{*},
\end{gathered}
$$

$N_{6}=\left(a_{11} c_{2} H_{2}-c_{1} \alpha_{1} H_{2}-c_{1} \rho_{1} H_{2}^{2} H_{1} Z^{*}\right) P_{1}^{*} P_{2}^{*} Z^{*}$. The Equation

(9) has at least one positive root say $\rho_{2}=\rho_{2}^{*}$.

Therefore, one pair of eigen values of the characteristic Equation (8) at $\rho_{2}=\rho_{2}^{*}$ are of the form $\lambda_{1,2}= \pm i v$, where $v$ is positive real number.

Now, we will verify the Hopf-bifurcation condition (iii), putting $\lambda_{\mathrm{i}}=u_{\mathrm{i}}+i v_{\mathrm{i}}$, in Equation (8), we get

$$
(u+i v)^{2}+A_{1}(u+i v)^{2}+A_{2}(u+i v)+A_{3}=0
$$

On separating the real and imaginary parts and eliminating $v$ between real and imaginary parts, we get

$$
8 u^{3}+8 A_{1} u^{2}+2\left(A_{1}^{2}+A_{2}\right) u+A_{1} A_{2}-A_{3}=0
$$

Again, differentiating (11) with respect to $\rho_{2}$, we have

$$
\begin{aligned}
& 24 u^{2} \frac{d u}{d \rho_{2}}+16 A_{1} u \frac{d u}{d \rho_{2}}+8 u^{2} \frac{d A_{1}}{d \rho_{2}} \\
& +2\left(A_{1}^{2}+A_{2}\right) \frac{d u}{d \rho_{2}}+2 u\left[2 A_{1} \frac{d A_{1}}{d \rho_{2}}+\frac{d A_{2}}{d \rho_{2}}\right]+\frac{d C}{d \rho_{2}}=0
\end{aligned}
$$

Now, since at $\rho_{2}=\rho_{2}^{*}, u\left(\rho_{2}^{*}\right)=0$ we get

$$
\left[\frac{d u}{d \rho_{2}}\right]_{\rho_{2}=\rho_{2}^{*}}=\frac{-\frac{d C}{d \rho_{2}}}{2\left(A_{1}^{2}+A_{2}\right)} \neq 0
$$

This ensures that the above system has a Hopf-bifurcation around the positive interior equilibrium $E^{*}$. At the Hopf bifurcation point, where the real parts of co mplex conjugate eigen values are zero, the roots of Equation (8) are $\lambda_{1,2}= \pm i v$, $\lambda_{3}=-\mathrm{A}_{1}$,

Where $v=\left|\sqrt{ } \mathrm{A}_{2}\right|$ and $A_{1}$ are to be evaluated at the bifurcation point. Next we seek a transformation matrix $P$ which reduces the matrix $A$ to the form

$$
P^{-1} A P=\left(\begin{array}{ccc}
0 & -v & 0 \\
v & 0 & 0 \\
0 & 0 & -A_{1}
\end{array}\right)
$$

where the non-singular matrix $\mathrm{P}$ is given as

Where

$$
P=\left(\begin{array}{lll}
m_{11} & m_{12} & m_{13} \\
m_{21} & m_{22} & m_{23} \\
m_{31} & m_{32} & m_{33}
\end{array}\right)
$$

$$
\begin{aligned}
m_{11} & =P_{1}^{*} P_{2}^{*}\left(a_{22} H_{1} \rho_{1}-H_{2} \alpha_{1} \rho_{2}\right), m_{12}=-v P_{1}^{*} H_{1} \rho_{1}, \\
m_{13} & =P_{1}^{*}\left(-A+P_{2}^{*} a_{22}\right) H_{1} \rho_{1}-P_{1}^{*} P_{2}^{*} H_{2} \alpha_{1} \rho_{2}, \\
m_{21} & =P_{1}^{*} P_{2}^{*}\left(-H_{1} \alpha_{2} \rho_{1}+a_{11} H_{2} \rho_{2}\right), \\
m_{22} & =-v P_{2}^{*} H_{2} \rho_{2}, \\
m_{23} & =-P_{1}^{*} P_{2}^{*} H_{1} \alpha_{2} \rho_{1}+P_{2}^{*}\left(-A+P_{2}^{*} a_{11}\right) H_{2} \rho_{2} \\
m_{31} & =v^{2}+P_{1}^{*} P_{2}^{*}\left(-a_{11} a_{22}+\alpha_{1} \alpha_{2}\right), \\
m_{32} & =v\left(P_{1}^{*} a_{11}+P_{2}^{*} a_{22}\right), \\
m_{32} & =-\left(P_{1}^{*} a_{11}-A_{1}\right)\left(-A_{2}+P_{2}^{*} a_{22}\right)+P_{1}^{*} P_{2}^{*} \alpha_{1} \alpha_{2} .
\end{aligned}
$$

To achieve normal form of the Equation (7), we make another change of variable i.e. $X=P Y$, where

$$
Y=\left(\begin{array}{l}
y_{1} \\
y_{2} \\
y_{3}
\end{array}\right)
$$

Through some a lgebraic man ipulations, Equation (7) takes the form

$$
\dot{Y}=\Omega Y+F
$$

where, $\Omega=P^{-1} A P$ and

$$
F=P^{-1} f=\left(\begin{array}{l}
F^{1}\left(y_{1}, y_{2}, y_{3}\right) \\
F^{2}\left(y_{1}, y_{2}, y_{3}\right) \\
F^{3}\left(y_{1}, y_{2}, y_{3}\right)
\end{array}\right)
$$

$\mathrm{f}$ is given by

$$
f=\left(\begin{array}{l}
f^{1}\left(y_{1}, y_{2}, y_{3}\right) \\
f^{2}\left(y_{1}, y_{2}, y_{3}\right) \\
f^{3}\left(y_{1}, y_{2}, y_{3}\right)
\end{array}\right)
$$

Where,

$$
\begin{aligned}
& f^{1}\left(y_{1}, y_{2}, y_{3}\right)=\left(m_{11} y_{1}+m_{12} y_{2}+m_{13} y_{3}\right)\left(b _ { 1 } \left(m_{11} y_{1}+m_{12} y_{2}\right.\right. \\
& \left.+m_{13} y_{3}\right)+b_{2}\left(m_{21} y_{1}+m_{22} y_{2}+m_{23} y_{3}\right)+b_{3}\left(m_{31} y_{1}+m_{32} y_{2}\right. \\
& \left.+m_{33} y_{3}\right)+b_{4}\left(m_{11} y_{1}+m_{12} y_{2}+m_{13} y_{3}\right)^{2}+b_{5}\left(m_{11} y_{1}+m_{12} y_{2}\right. \\
& \left.+m_{13} y_{3}\right)\left(m_{31} y_{1}+m_{32} y_{2}+m_{33} y_{3}\right), \\
& f^{2}\left(y_{1}, y_{2}, y_{3}\right)=\left(m_{21} y_{1}+m_{22} y_{2}+m_{23} y_{3}\right)\left(n _ { 1 } \left(m_{21} y_{1}+m_{22} y_{2}\right.\right. \\
& \left.+m_{23} y_{3}\right)+n_{2}\left(m_{11} y_{1}+m_{12} y_{2}+m_{13} y_{3}\right)+n_{3}\left(m_{31} y_{1}+m_{32} y_{2}\right. \\
& \left.+m_{33} y_{3}\right)+n_{4}\left(m_{21} y_{1}+m_{22} y_{2}+m_{23} y_{3}\right)^{2}+n_{5}\left(m_{21} y_{1}+m_{22} y_{2}\right. \\
& \left.\left.+m_{23} y_{3}\right)\left(m_{31} y_{1}+m_{32} y_{2}+m_{33} y_{3}\right)\right),
\end{aligned}
$$




$$
\begin{aligned}
& f^{3}\left(y_{1}, y_{2}, y_{3}\right)=\left(m_{11} y_{1}+m_{12} y_{2}+m_{13} y_{3}\right)\left(q _ { 1 } \left(m_{11} y_{1}+m_{12} y_{2}\right.\right. \\
& \left.+m_{13} y_{3}\right)+q_{2}\left(m_{11} y_{1}+m_{12} y_{2}+m_{13} y_{3}\right)^{2}+q_{3}\left(m_{31} y_{1}+m_{32} y_{2}\right. \\
& \left.+m_{33} y_{3}\right)+q_{4}\left(m_{11} y_{1}+m_{12} y_{2}+m_{13} y_{3}\right)\left(m_{31} y_{1}+m_{32} y_{2}+m_{33} y_{3}\right) \\
& +\left(m_{21} y_{1}+m_{22} y_{2}+m_{23} y_{3}\right)\left(q_{5}\left(m_{11} y_{1}+m_{12} y_{2}+m_{13} y_{3}\right)+q_{6}\right. \\
& \left(m_{21} y_{1}+m_{22} y_{2}+m_{23} y_{3}\right)^{2}+q_{7}\left(m_{31} y_{1}+m_{32} y_{2}+m_{33} y_{3}\right)+ \\
& q_{8}\left(m_{21} y_{1}+m_{22} y_{2}+m_{23} y_{3}\right) \\
& \left.\left(m_{31} y_{1}+m_{32} y_{2}+m_{33} y_{3}\right)\right) .
\end{aligned}
$$

Equation (13) is the normal form of Equation (7) from which the stability and direction of the Hopf bifurcation can be computed. In Equation (13), on the right hand side of the first term is linear and the second is non-linear in y's. From these non-linear terms the stability and direction of the Hopf bifurcation is obtained[25].

$$
g_{11}=\frac{1}{4}\left[\frac{\partial^{2} F^{1}}{\partial y_{1}^{2}}+\frac{\partial^{2} F^{1}}{\partial y_{2}^{2}}+i\left(\frac{\partial^{2} F^{2}}{\partial y_{1}^{2}}+\frac{\partial^{2} F^{2}}{\partial y_{2}^{2}}\right)\right]
$$

$g_{02}=\frac{1}{4}\left[\frac{\partial^{2} F^{1}}{\partial y_{1}^{2}}-\frac{\partial^{2} F^{1}}{\partial y_{2}^{2}}-2 \frac{\partial^{2} F^{2}}{\partial y_{1} \partial y_{2}}+i\left(\frac{\partial^{2} F^{2}}{\partial y_{1}^{2}}-\frac{\partial^{2} F^{2}}{\partial y_{2}^{2}}+2 \frac{\partial^{2} F^{1}}{\partial y_{1} \partial y_{2}}\right)\right]$

$$
\begin{gathered}
g_{20}=\frac{1}{4}\left[\frac{\partial^{2} F^{1}}{\partial y_{1}^{2}}-\frac{\partial^{2} F^{1}}{\partial y_{2}^{2}}+2 \frac{\partial^{2} F^{2}}{\partial y_{1} \partial y_{2}}+i\left(\frac{\partial^{2} F^{2}}{\partial y_{1}^{2}}-\frac{\partial^{2} F^{2}}{\partial y_{2}^{2}}-2 \frac{\partial^{2} F^{1}}{\partial y_{1} \partial y_{2}}\right)\right] \\
g_{21}=G_{21}+2 G_{101} w_{11}+G_{101} w_{20},
\end{gathered}
$$

where,

$$
\begin{gathered}
G_{21}=\frac{1}{8}\left[\frac{\partial^{3} F^{1}}{\partial y_{1}^{3}}+\frac{\partial^{3} F^{1}}{\partial y_{1} \partial y_{2}}+\frac{\partial^{3} F^{2}}{\partial y_{1}^{2} \partial y_{2}}+\frac{\partial^{3} F^{2}}{\partial y_{2}^{3}}\right. \\
\left.+i\left(\frac{\partial^{3} F^{2}}{\partial y_{1}^{3}}+\frac{\partial^{3} F^{2}}{\partial y_{1} \partial y_{2}^{2}}-\frac{\partial^{3} F^{1}}{\partial y_{1}^{2} \partial y_{2}}-\frac{\partial^{3} F^{1}}{\partial y_{2}^{3}}\right)\right] \\
G_{110}=\frac{1}{2}\left[\frac{\partial^{2} F^{1}}{\partial y_{1} \partial y_{3}}+\frac{\partial^{2} F^{2}}{\partial y_{2} \partial y_{3}}+i\left(\frac{\partial^{2} F^{2}}{\partial y_{1} \partial y_{3}}-\frac{\partial^{2} F^{1}}{\partial y_{2} \partial y_{3}}\right)\right], \\
G_{101}=\frac{1}{2}\left[\frac{\partial^{2} F^{1}}{\partial y_{1} \partial y_{3}}-\frac{\partial^{2} F^{2}}{\partial y_{2} \partial y_{3}}+i\left(\frac{\partial^{2} F^{2}}{\partial y_{1} \partial y_{3}}+\frac{\partial^{2} F^{1}}{\partial y_{2} \partial y_{3}}\right)\right], \\
h_{11}=\frac{1}{4}\left[\frac{\partial^{2} F^{3}}{\partial y_{1}^{2}}+\frac{\partial^{2} F^{3}}{\partial y_{2}^{2}}\right], \\
h_{20}=\frac{1}{4}\left[\frac{\partial^{2} F^{3}}{\partial y_{1}^{2}}-\frac{\partial^{2} F^{3}}{\partial y_{2}^{2}}-2 i \frac{\partial^{2} F^{1}}{\partial y_{1} \partial y_{2}}\right], \\
w_{11}=\frac{h_{11}}{A_{1}}, \\
w_{20}=\frac{h_{20}}{\left(A_{1}+2 i v\right)} .
\end{gathered}
$$

All these partial derivatives are determined at the Hopf bifurcation point $\rho_{2}$ as well as at the origin. Based on the above analysis, we can see that each $g_{i j}$ can be determined by the parameters. Thus we can compute the following quantities:

$$
\begin{gathered}
C_{1}(0)=\frac{i}{2 v \rho_{2}^{*}}\left(g_{11} g_{20}-2\left|g_{11}\right|^{2}-\frac{\left|g_{02}\right|^{2}}{3}\right)+\frac{g_{21}}{2}, \\
\mu_{2}=\frac{\operatorname{Re}\left\{C_{1}(0)\right\}}{\operatorname{Re}\left\{\lambda^{\prime}\left(\rho_{2}^{*}\right)\right\}},
\end{gathered}
$$

$$
\begin{array}{r}
\beta_{2}=2 \operatorname{Re}\left\{C_{1}(0)\right\}, \\
T_{2}=-\frac{\operatorname{Im}\left\{C_{1}(0)\right\}+\mu_{2} \operatorname{Im}\left\{\lambda^{\prime}\left(\rho_{2}^{*}\right)\right\}}{v},
\end{array}
$$

The orem 3.1. $\mu_{2}$ determines the direction of the Hopf bifurcation: if $\mu_{2}>0\left(\mu_{2}<0\right)$, then the Hopf bifurcation is supercritical (subcritical) and the bifurcating periodic solutions exist for $\rho_{2}>\rho_{2} *\left(\rho_{2}<\rho_{2} *\right) ; \beta_{2}$ determines the stability of bifurcating periodic solutions. and $T_{2}$ determines the period of the bifurcating periodic solutions, the period increases (decreases) if $T_{2}>0\left(T_{2}<0\right)$.

Now we will study the Hopf-bifurcation $[25,11,26]$ of the system without delay taking $\rho_{1}$ as the bifurcation parameter. The necessary and sufficient conditions for the existence of the Hopf-bifurcation for $\rho_{1}=\rho_{1}{ }^{*}$, if it exist are (i) $A_{\mathrm{i}}\left(\rho_{1}{ }^{*}\right)>0$, $\mathrm{i}=1,2,3$ (ii) $A_{1}\left(\rho_{1}^{*}\right) A_{2}\left(\rho_{1}^{*}\right)-A_{3}\left(\rho_{1}^{*}\right)=0$ and (iii) the eigenvalues of the characteristic Equation should be of the form $\lambda_{\mathrm{i}}=u_{\mathrm{i}}+i v_{\mathrm{i}}$, and $\frac{d u}{d \rho_{1}} \neq 0, \mathrm{i}=1,2,3$. After substituting the values, the condition $C=A_{1} A_{2}-A_{3}$ becomes

$$
-B_{4} \rho_{1}^{2}+B_{5} \rho_{1}+B_{6}=0
$$

Where,

$$
\begin{gathered}
B_{4}=M_{2} M_{4}, B_{5}=M_{1} M_{4}-M_{2} M_{3}-M_{6}, B_{6}=M_{1} M_{3}-M_{5} \\
M_{1}=a_{22} P_{2}^{*}+\frac{r_{1}}{k} P_{1}^{*}, M_{2}=Z H_{1}^{2} P_{1}^{*}, \\
M_{3}=\left(a_{22} \frac{r_{1}}{k} \alpha_{1} \alpha_{2}\right) P_{1}^{*} P_{2}^{*}+c_{2} \rho_{2} H_{2} P_{2}^{*} Z^{*}, \\
M_{4}=c_{1} H_{1} P_{1}^{*} Z^{*}-a_{22} P_{1}^{*} P_{2}^{*} Z^{*} H_{1}^{2}, \\
M_{5}=\left(\frac{r_{1}}{k} c_{2} \rho_{2} H_{2}-c_{1} \rho_{2} H_{2} \alpha_{1}\right) P_{1}^{*} P_{2}^{*} Z^{*}, \\
M_{6}=\left(a_{22} c_{1} H_{1}-c_{2} \alpha_{2} H_{1}-c_{2} \rho_{2} H_{1}^{2} H_{2} Z^{*}\right.
\end{gathered}
$$

The Equation (15) has at least one positive root say $\rho_{1}=$ $\rho_{1}$ * Therefore, one pair of eigenvalues of the characteristic Equation (8) at $\rho_{1}=\rho_{1} *$ are of the form $\lambda_{1,2}= \pm i v$, where $v$ is positive real number. Now, we will verify the Hopf-bifurcation condition (iii), putting $\lambda=u+i v$ in (8), we get

$$
(u+i v)^{3}+A_{1}(u+i v)^{2}+A_{2}(u+i v)+A_{1}=0 .
$$

On separating the real and imaginary parts and eliminating $v$ between real and imaginary parts, we get

$$
8 u^{3}+8 u^{2}+2\left(A_{1}^{2}+A_{2}\right) u+A_{1} A_{2}-A_{3}=0
$$

Again, differentiating (17) with respect to $\rho_{1}$, we have

$$
\begin{aligned}
& 24 u^{2} \frac{d u}{d \rho_{1}}+16 A_{1} u \frac{d u}{d \rho_{1}}+8 u^{2} \frac{d A_{1}}{d \rho_{1}} \\
& +2\left(A_{1}^{2}+A_{2}\right) \frac{d u}{d \rho_{1}}+\left[2 A_{1} \frac{d A_{1}}{d \rho_{1}}+\frac{d A_{2}}{d \rho_{1}}\right]+\frac{d C}{d \rho_{1}}=0
\end{aligned}
$$

Now, since at $\rho_{1}=\rho_{1}^{*}, u\left(\rho_{1}^{*}\right)=0$ we get

$$
\left[\frac{d u}{d \rho_{1}}\right]_{\rho_{1}=\rho_{1}^{*}}=\frac{-\frac{d C}{d \rho_{1}}}{2\left(A_{1}^{2}+A_{2}\right)} \neq 0
$$


This ensures that the above system has a Hopf-bifurcation around the positive interior equilibrium $E^{*}$.

As above, similarly we have computed $C_{1}(0), \mu_{2}, \beta_{2}$ and $T_{2}$ for studying the direction and stability of Hopf-bifurcating solution for the parameter $\rho_{1}$ and obtain,

$$
\begin{gathered}
C_{1}(0)=\frac{i}{2 v \rho_{1}^{*}}\left(g_{11} g_{20}-2\left|g_{11}\right|^{2}-\frac{\left|g_{02}\right|^{2}}{3}\right)+\frac{g_{21}}{2}, \\
\mu_{2}=\frac{\operatorname{Re}\left\{C_{1}(0)\right\}}{\operatorname{Re}\left\{\lambda^{\prime}\left(\rho_{1}^{*}\right)\right\}}, \\
\beta_{2}=2 \operatorname{Re}\left\{C_{1}(0)\right\}, \\
T_{2}=-\frac{\operatorname{Im}\left\{C_{1}(0)\right\}+\mu_{2} \operatorname{Im}\left\{\lambda^{\prime}\left(\rho_{1}^{*}\right)\right\}}{v} .
\end{gathered}
$$

Theorem 3.2. $\mu_{2}$ determines the direction of the Hopf bifurcation: if $\mu_{2}>0\left(\mu_{2}<0\right)$, then the Hopf bifurcation is supercritical (subcritical) and the bifurcating periodic solutions exist for $\rho_{1}>\rho_{1}^{*}\left(\rho_{1}<\rho_{1} *\right) ; \beta_{2}$ determines the stability of bifurcating periodic solutions. and $T_{2}$ determines the period of the bifurcating periodic solutions, the period increases (decreases) if $T_{2}>0\left(T_{2}<0\right)$.

Now we will study the Hopf-bifurcation[25, 11, 26] of the system without delay taking $h_{1}$ as the bifurcation parameter. The necessary and sufficient conditions for the existence of the Hopf-bifurcation for $h_{1}=h_{1}^{*}$, if it exist are (i) $A_{\mathrm{i}}\left(h_{1}^{*}\right)>0$, $\mathrm{i}=1,2,3$ (ii) $A_{1}\left(h_{1}^{*}\right) A_{2}\left(h_{1}^{*}\right)-A_{3}\left(h_{1}^{*}\right)=0$ and (iii) the eigenvalues of the characteristic equation should be of the form $\lambda_{i}=u_{i}+i v_{i}$ and $d u / d h_{1} \neq 0, \mathrm{i}=1,2,3$. After substituting the values, the condition $C=A_{1} A_{2}-A_{3}$ becomes

$$
a_{1} h_{1}^{5}+a_{2} h_{1}^{4}+a_{3} h_{1}^{3}+a_{4} h_{1}^{2}+a_{5} h_{1}+a_{6}=0,
$$

Where,

$$
\begin{aligned}
& a_{1}=e_{1} e_{3}-e_{6}, a_{2}=5 P_{1}^{*}\left(e_{1} e_{3}-e_{6}\right)+e_{7}, \\
& a_{3}=P_{1}^{* 2}\left(6 e_{1} e_{3}-10 e_{6}\right)+2 P_{1}^{*} e_{7}+e_{2} e_{3}+e_{1} e_{4}-e_{8}, \\
& a_{4}=-9 e_{6} P_{1}^{* 3}+P_{1}^{* 2} e_{7}+P_{1}^{*}\left(2 e_{1} e_{4}-2 e_{8}+3 e_{2} e_{3}\right)+e_{1} e_{5}+e_{9}, \\
& a_{5}=e_{2} e_{4}+2 e_{5} e_{1} P_{1}^{*}+2 e_{9} P_{1}^{*}\left(-e_{8}-3 e_{6} P_{1}^{* 2}\right), \\
& a_{6}=e_{5} e_{2}+P_{1}^{* 2} e_{9}, \\
& e_{1}=\left(\frac{r_{1} P_{1}^{*}}{k}+a_{22} P_{2}\right), e_{2}=e_{1} P_{1}^{* 2}-Z^{*} P_{1}^{*} \rho_{1}, \\
& e_{3}=\left(\frac{r_{1} a_{22}}{k}-\alpha_{1} \alpha_{2}\right) P_{1}^{*} P_{2}^{*}+c_{2} \rho_{2} H_{2} P_{2}^{*} Z^{*}, \\
& e_{4}=3 P_{1}^{* 2}-\rho_{1} a_{22} Z^{*} P_{1}^{* 2} P_{2}^{*}+\left(\gamma_{1}-\theta_{1}\right) \rho_{1} P_{1}^{*} Z^{*}, \\
& e_{5}=e_{3} P_{1}^{* 3} \rho_{1} a_{22} Z^{*} P_{1}^{* 2}, \\
& e_{6}=\frac{r_{1} c_{2} \rho_{2} H_{2} P_{1}^{*} P_{2}^{*} Z^{*}}{k}, \\
& e_{7}=P_{1}^{*} P_{2}^{*} Z^{*}\left(\left(\gamma_{1}-\theta_{1}\right) \rho_{2} H_{2} \alpha_{1} \rho_{1} \alpha_{2}\right) \\
& e_{8}=P_{1}^{*} P_{2}^{*} Z^{*}\left(a_{22} \rho_{1}\left(\gamma_{1}-\theta_{1}\right)-P_{1}^{*}\left(\gamma_{1}-\theta_{1}\right) \rho_{2} H_{2} \alpha_{1}-2 c_{2} \rho_{1} \alpha_{2} P_{1}^{*}\right), \\
& e_{9}=P_{1}^{* 3}\left(c_{2} \rho_{1} \alpha_{2} P_{2}^{*} Z^{*}-e_{6}\right) .
\end{aligned}
$$

The Equation (19) has at least one positive root say $h_{1}=$ $h_{1}^{*}$ Therefore, one pair of eigenvalues of the characteristic Equation (8) at $h_{1}=h_{1}^{*}$ are of the form $\lambda_{1,2}=\neq \pm i \mathrm{v}$, where $v$ is positive real nu mber.

Now, we will verify the Hopf-bifurcation condition (iii), putting $\lambda=u+i v$ in (8), we get

$$
(u+i v)^{3}+A_{1}(u+i v)+A_{2}(u+i v) A_{3}+A_{3}=0
$$

On separating the real and imaginary parts and eliminating $v$ between real and imaginary parts, we get

$$
8 u^{3}+8 A_{1} u^{2}+2\left(A_{1}^{2}+A_{2}\right) u+A_{1} A_{2}-A_{3}=0
$$

Again, differentiating (21) with respect to $h_{1}$, we have

$$
\begin{aligned}
& 24 u^{2} \frac{d u}{d h_{1}}+16 u \frac{d u}{d h_{1}}+8 u^{2} \frac{d A_{1}}{d h_{1}} 2\left(A_{1}^{2}\right. \\
& \left.+A_{2}\right) \frac{d u}{d h_{1}}+2 u\left[2 A_{1} \frac{d A_{1}}{d h_{1}}+\frac{d A_{2}}{d h_{1}}\right]+\frac{d C}{d h_{1}}=0
\end{aligned}
$$

Now, since at $h_{1}=h_{1}^{*}, u\left(h_{1}^{*},\right)=0$ we get

$$
\left[\frac{d u}{d h_{1}}\right]_{h_{1}=h_{1}^{*}}=\frac{-\frac{d C}{d h_{1}}}{2\left(A_{1}^{2}+A_{2}\right)} \neq 0
$$

This ensures that the above system has a Hopf-bifurcation around the positive interior equilibrium $E^{*}$.

As above, similarly we have computed $C_{1}(0), \mu_{2}, \beta_{2}$ and $\mathrm{T}_{2}$ for studying the direction and stability of Hopf-bifurcating solution for the parameter $\mathrm{h}_{1}$ and obtain,

$$
\begin{gathered}
C_{1}(0)=\frac{i}{2 v h_{1}^{*}}\left(g_{11} g_{20}-2\left|g_{11}\right|^{2}-\frac{\left|g_{02}\right|^{2}}{3}\right)+\frac{g_{21}}{2}, \\
\mu_{2}=\frac{\operatorname{Re}\left\{C_{1}(0)\right\}}{\operatorname{Re}\left\{\lambda^{\prime}\left(h_{1}^{*}\right)\right\}}, \\
\beta_{2}=2 \operatorname{Re}\left\{C_{1}(0)\right\}, \\
T_{2}=-\frac{\operatorname{Im}\left\{C_{1}(0)\right\}+\mu_{2} \operatorname{Im}\left\{\lambda^{\prime}\left(h_{1}^{*}\right)\right\}}{v} .
\end{gathered}
$$

Theorem 3.3. $\mu_{2}$ determines the direction of the Hopf bifurcation: if $\mu_{2}>0\left(\mu_{2}<0\right)$, then the Hopf bifurcation is supercritical (subcritical) and the bifurcating periodic solutions exist for $h_{1}>h_{1}^{*}\left(h_{1}<h_{1}^{*}\right) ; \beta_{2}$ determines the stability of bifurcating periodic solutions. and $T_{2}$ determines the period of the bifurcating periodic solutions, the period increases (decreases) if $T_{2}>0\left(T_{2}<0\right)$.

Similarly if we take $h_{2}$ as the bifurcation parameter. The necessary and sufficient conditions for the existence of the Hopf-bifurcation for $h_{2}=h_{2} *$, if it exist are (i) $A_{\mathrm{i}}\left(h_{2} *\right)>0$, i=1, 2, 3 (ii) $A_{1}\left(h_{2} *\right) A_{2}\left(h_{2}^{*}\right)-A_{3}\left(h_{2} *\right)=0$ and (iii) the eigenvalues of the characteristic equation should be of the form $\lambda_{\mathrm{i}}=u_{\mathrm{i}}+i v_{\mathrm{i}}$, and $d u / d h_{2} \neq 0, \mathrm{i}=1,2,3$. After substituting the values, the condition $C=A_{1} A_{2}-A_{3}$ becomes

$$
L_{1} h_{2}^{5}+L_{2} h_{2}^{4}+L_{3} h_{2}^{3}+L_{4} h_{2}^{2}+L_{5} h_{2}+L_{6}=0,
$$

Where,

$$
\begin{aligned}
& L_{1}=l_{1} l_{2}-l_{6}, L_{2}=5 P_{2}^{*}\left(l_{1} l_{3}-l_{6}\right)+l_{7}, \\
& L_{3}=P_{2}^{* 2}\left(6 l_{1} l_{3}-10 l_{6}\right)+2 P_{2}^{*} l_{7}+l_{2} l_{3}+l_{1} l_{4}-l_{8}, \\
& L_{4}=-9 l_{6} P_{2}^{* 3}+P_{2}^{* 2} l_{7}+P_{2}^{*}\left(2 l_{1} l_{4}-2 l_{8}+3 l_{2} l_{3}\right)+l_{1} l_{5}+l_{9}, \\
& L_{5}=l_{2} l_{4}+2 l_{5} l_{1} P_{2}^{*}+2 l_{9} P_{2}^{*}+P_{2}^{* 2}\left(-l_{8}-3 l_{6} P_{2}^{* 2}\right), \\
& L_{4}=-9 l_{6} P_{2}^{* 3}+P_{2}^{* 2} l_{7}+P_{2}^{*}\left(2 l_{1} l_{4}-2 l_{8}+3 l_{2} l_{3}\right)+l_{1} l_{5}+l_{9}, \\
& L_{3}=P_{2}^{* 2}\left(6 l_{1} l_{3}-10 l_{6}\right)+2 P_{2}^{*} l_{7}+l_{2} l_{3}+l_{1} l_{4}-l_{8},
\end{aligned}
$$




$$
\begin{aligned}
& l_{1}=\left(\frac{r_{2} P_{2}^{*}}{k}+a_{11} P_{1}\right), \\
& l_{2}=3 l_{1} P_{2}^{* 2}-Z^{*} P_{2}^{*} \rho_{2}, \\
& l_{3}=\left(\frac{r_{2} a_{11}}{k}-\alpha_{1} \alpha_{2}\right) P_{1}^{*} P_{2}^{*}+c_{1} \rho_{1} H_{1} P_{1}^{*} Z^{*}, \\
& l_{4}=3 l_{3} P_{2}^{* 2}-\rho_{2} a_{11} Z^{*} P_{2}^{* 2} P_{1}^{*}+\left(\gamma_{2}-\theta_{2}\right) \rho_{2} P_{2}^{*} Z^{*}, \\
& l_{5}=l_{3} P_{2}^{* 3}-\rho_{2} a_{11} Z^{*} P_{2}^{* 2} P_{1}^{*}, \\
& l_{6}=\frac{r_{2} c_{2} \rho_{1} H_{1} P_{1}^{*} P_{2}^{*} Z^{*}}{k}, \\
& l_{7}=P_{1}^{*} P_{2}^{*} Z^{*}\left(\left(\gamma_{2}-\theta_{2}\right) \rho_{1} H_{1} \alpha_{2}+c_{1} \rho_{2} \alpha_{1}\right), \\
& l_{8}=P_{1}^{*} P_{2}^{*} Z^{*}\left(a_{11} \rho_{2}\left(\gamma_{2}-\theta_{2}\right) \rho_{1} H_{1} \alpha_{2}-2 c_{1} \rho_{2} \alpha_{1} P_{2}^{*}\right), \\
& l_{9}=P_{2}^{* 3}\left(c_{1} \rho_{2} \alpha_{1} P_{1}^{*} Z^{*}-l_{6}\right) .
\end{aligned}
$$

The Equation (23) has at least one positive root say $h_{2}=$ $h_{2}{ }^{*}$. Therefore, one pair of eigenvalues of the characteristic Equation (8) at $h_{2}=h_{2} *$ are of the form $\lambda_{1,2}= \pm i v$, where $v$ is positive real nu mber and also

$$
\left[\frac{d u}{d h_{2}}\right]_{h_{2}=h_{2}^{*}}=\frac{-\frac{d C}{d h_{2}}}{2\left(A_{1}^{2}+A_{2}\right)} \neq 0
$$

This ensures that the above system has a Hopf-bifurcation around the positive interior equilibrium $E^{*}$.

As above, similarly we have computed $C_{1}(0), \mu_{2}, \beta_{2}$ and $\mathrm{T}_{2}$ for studying the direction and stability of Hopf-bifurcating solution for the parameter $h_{2}$ and obtain,

$$
\begin{gathered}
C_{1}(0)=\frac{i}{2 v h_{2}^{*}}\left(g_{11} g_{20}-2\left|g_{11}\right|^{2}-\frac{\left|g_{02}\right|^{2}}{3}\right)+\frac{g_{21}}{2}, \\
\mu_{2}=\frac{\operatorname{Re}\left\{C_{1}(0)\right\}}{\operatorname{Re}\left\{\lambda^{\prime}\left(h_{2}^{*}\right)\right\}}, \\
\beta_{2}=2 \operatorname{Re}\left\{C_{1}(0)\right\}, \\
T_{2}=-\frac{\operatorname{Im}\left\{C_{1}(0)\right\}+\mu_{2} \operatorname{Im}\left\{\lambda^{\prime}\left(h_{2}^{*}\right)\right\}}{v} .
\end{gathered}
$$

Theorem 3.4. $\mu_{2}$ determines the direction of the Hopf bifurcation: if $\mu_{2}>0\left(\mu_{2}<0\right)$, then the Hopf bifurcation is supercritical (subcritical) and the bifurcating periodic solutions exist for $h_{2}>h_{2}^{*}\left(h_{2}<h_{2}^{*}\right) ; \beta_{2}$ determines the stability of bifurcating periodic solutions. and $T_{2}$ determines the period of the bifurcating periodic solutions, the period increases (decreases) if $T_{2}>0\left(T_{2}<0\right)$.

\section{Stability of the Interior Equilibrium and Local Hopf Bifurcations of the Model with Time Delay}

From the previous analysis we know that this system has seven equilibriums with certain conditions. In this section, we shall study the stability of the interior equilibrium and the existence of local Hopf bifurcations because the remaining six equilibria do not show the effects of delay in the analys is process. The characteristic equation of system of Equations (1)-(3) at the equilibrium $E^{*}$ is

$$
\lambda^{3}+M_{1} \lambda^{2}+M_{2} \lambda+M_{3}+e^{-\lambda \tau}\left(N_{1} \lambda^{2}+N_{2} \lambda+N_{3}\right)=0
$$

Where,

$$
\begin{aligned}
& M_{1}=A_{1}-N_{1}, N_{1}=\theta_{1} P_{1}^{*} H_{1}+\theta_{2} P_{2}^{*} H_{2}, \\
& M_{2}=P_{1}^{*}\left(-a_{11} c_{7}+P_{2}^{*}\left(a_{11} a_{22}-\alpha_{1} \alpha_{2}\right)+\right. \\
& \left.Z^{*} h_{1} H_{1}^{3} \gamma_{1} \rho_{1}\right)+P_{2}^{*}\left(-a_{22} c_{7}-Z^{*} h_{2} H_{2}^{3} \theta_{2} \rho_{2}\right), \\
& N_{2}=P_{1}^{*}\left(a_{11} c_{7}-Z^{*} h_{1} H_{1}^{3} \theta_{1} \rho_{1}\right)+P_{2}^{*}\left(a_{22} c_{7}-Z^{*} h_{2} H_{2}^{3} \theta_{2} \rho_{2}\right), \\
& M_{3}=P_{1}^{*} P_{2}^{*}\left(c_{7} \alpha_{1} \alpha_{2}+a_{11}\left(-a_{22} c_{7}+Z^{*} h_{2} H_{2}^{3} \gamma_{2} \rho_{2}\right)\right. \\
& \left.+Z^{*} h_{1}\left(a_{22} h_{1} H_{1}^{2} \gamma_{1} \rho_{1}-H_{2}\left(h_{2} H_{2} \alpha_{2} \gamma_{2} \rho_{1}+h_{1} H_{1} \alpha_{1} \gamma_{1} \rho_{2}\right)\right)\right), \\
& N_{3}=P_{1}^{*} P_{2}^{*}\left(-c_{7} \alpha_{1} \alpha_{2}+Z^{*} H_{1}\left(-a_{22} h_{1} H_{1}^{2} \theta_{1} \rho_{1}+h_{2} H_{2}^{2} \alpha_{2} \theta_{2} \rho_{1}\right.\right. \\
& \left.\left.+h_{1} H_{1} H_{2} \alpha_{1} \theta_{1} \rho_{2}\right)+a_{11}\left(a_{22} c_{7}-Z^{*} h_{2} H_{2}^{3} \theta_{2} \rho_{2}\right)\right), \\
& c_{7}=\theta_{1} P_{1}^{*} H_{1}+\theta_{2} P_{2}^{*} H_{2} .
\end{aligned}
$$

For stability of $E^{*}$, all the eigenvalues of the characteristic Equation (25) should have negative real part. It is difficult to analyze the condition under which Equation (25) has all roots with negative real part. However, for zero time delay, Equation (25) beco mes

$$
\lambda^{3}+\left(M_{1}+N_{1}\right) \lambda^{2}+\left(M_{2}+N_{2}\right) \lambda+\left(M_{3}+N_{3}\right)=0(26)
$$

By Routh-Hurwitz criterion

$$
\left(M_{3}+N_{3}\right)>0,\left(M_{1}+N_{1}\right)\left(M_{2}+N_{2}\right)>\left(M_{3}+N_{3}\right) .
$$

then all roots of Equation (26) have negative real parts.

Obviously, $i \mathrm{w}(\tau)(\mathrm{w}>0)$ is a root of Equation (26) if and only if

$$
\begin{aligned}
& -i w^{3}-M_{1} w^{2}+i M_{2} w+M_{3}+(\cos w \tau \\
& -i \sin w \tau)\left(-N_{1} w+i N_{2} w+N_{3}\right)=0
\end{aligned}
$$

Separating the real and imaginary parts,

$$
\begin{gathered}
M_{3}-M_{1} w^{2}+\left(N_{3}-N_{1} w^{2}\right) \cos w \tau+N_{2} w \sin w \tau=0 \\
M_{2} w-w^{3}+N_{2} w \cos w \tau-\left(N_{3}-N_{1} w^{2}\right) \sin w \tau=0
\end{gathered}
$$

which leads to

$$
w^{6}+p w^{4}+q w^{2}+r=0,
$$

where

$$
\begin{aligned}
& P=M_{1}^{2}-N_{1}^{2}-2 M_{2}, \\
& q=M_{2}^{2}-N_{2}^{2}+2 N_{1} N_{3}-2 M_{1} M_{3}, \\
& r=M_{3}^{2}-N_{2}^{3} .
\end{aligned}
$$

Let $x=w^{2}$, then Equation (30) becomes

$$
x^{3}+p x^{2}+q x+r=0
$$

and $h(x)=x^{3}+p x^{2}+q x+r$

Lemma 4.1. For the polynomial Equation (31), we have the following results.

(1) If $r<0$, then Equation (31) has at least one positive root.

(2) If $r \geq 0$ and $\left(p^{2}-3 q\right) \leq 0$, then Equation (31) has no positive roots.

(3) If $r \geq 0$ and $\Delta>0$, then Equation (31) has positive roots 
if and only if $x_{1}^{*}=\frac{-p+\sqrt{\left(p^{2}-3 q\right)}}{3}>0$ and $h\left(x_{1}^{*}\right) \leq 0$

The proof of Lemma 4.1 is similar to that in the proof of Lemma 2.1 in[7], we therefore o mit it here.

From Equation (28) and Equation (29), we obtain,

$\cos w \tau=\frac{-\left(N_{2} w^{2} M_{2}^{2}+\left(M_{3}-M_{1} w^{2}\right)\left(N_{3}-N_{1} w^{2}\right)\right)}{\left(N_{3}-N_{1} w^{2}\right)^{2}+\left(N_{2} w\right)^{2}}$

$\tau_{0}=\frac{1}{w} \arccos \left(\frac{-\left(N_{2} w^{2} M_{2}^{2}+\left(M_{3}-M_{1} w^{2}\right)\left(N_{3}-N_{1} w^{2}\right)\right)}{\left(N_{3}-N_{1} w^{2}\right)^{2}+\left(N_{2} w\right)^{2}}+2 j \pi\right)(33)$

Whemm $-4,2$ Suppose that $x_{0}=w^{2}$. Then the sign of $\alpha\left(\tau_{0}\right)$ is coincident with that of $h\left(x_{0}\right)$.

Proof. Let $\lambda=\lambda(\tau)$ be the root of Equation (25). Substituting $\lambda(\tau)$ into Equation (25) and differentiating both side of Equation (25) with respect to $\tau$, it fo llows that

$$
\begin{aligned}
& {\left[\left(3 \lambda^{2}+2 M_{1} \lambda+M_{2}\right)\right] \frac{d \lambda}{d \tau}+\left(\left(\lambda^{2} N_{1}+\lambda N_{2}+N_{3}\right)(-\tau)\right.} \\
& \left.+\left(2 \lambda N_{1}+N_{2}\right)\right) e^{-\lambda \tau} \frac{d \lambda}{d \tau}=\lambda\left(\lambda^{2} N_{1}+\lambda N_{2}+N_{3}\right) e^{-\lambda \tau}
\end{aligned}
$$

Thus

$\left(\frac{d \lambda}{d \tau}\right)^{-1}=\frac{\left(3 \lambda^{2}+2 M_{1} \lambda+M_{2}\right) e^{-\lambda \tau}}{\lambda\left(\lambda N_{1}^{2}+\lambda N_{2}+N_{3}\right)}+\frac{\left(2 \lambda N_{1}+N_{2}\right)}{\lambda\left(\lambda N_{1}^{2}+\lambda N_{2}+N_{3}\right)}-\frac{\tau}{\lambda}$

From (28)-(30), we have

$$
\begin{gathered}
\alpha^{\prime}\left(\tau_{0}\right)=\operatorname{Re}\left[\frac{\left(3 \lambda^{2}+2 M_{1} \lambda+M_{2}\right) e^{-\lambda \tau}}{\lambda\left(\lambda N_{1}^{2}+\lambda N_{2}+N_{3}\right)}\right]+\operatorname{Re}\left[\frac{\left(2 \lambda N_{1}+N_{2}\right)}{\lambda\left(\lambda N_{1}^{2}+\lambda N_{2}+N_{3}\right)}\right] \\
=\frac{1}{\Lambda}\left[3 w_{0}^{6}+2\left(M_{1}^{2}-N_{1}^{2}-2 M_{2}\right) w_{0}^{4}+\left(M_{2}^{2}-N_{2}^{2}+2 N_{1} N_{2}-2 M_{1} M_{2}\right) w_{0}^{2}\right] \\
=\frac{1}{\Lambda}\left(3 w_{0}^{6}+2 p w_{0}^{2}+q w_{0}^{2}\right)=\frac{w_{0}}{\Lambda} h^{\prime}\left(w_{0}\right),
\end{gathered}
$$

where $\Lambda=B_{2}^{2} W_{0}^{2}+C_{2}^{2}$. Notice that $\Lambda>0$ and $w_{0}>0$, we conclude that $\operatorname{sign}\left[\alpha^{\prime}\left(\tau_{0}\right)\right]=\operatorname{sign}\left[h^{\prime}\left(w_{0}\right)\right]$. This proves the lemma.

The ore $\mathbf{m} 5.1$ Suppose that $(H 1)$ is satisfied

(i) If $r \geq 0$ and $\Delta \leq 0$, all roots of Equation (25) have negative real parts for all $\tau \geq 0$, and hence the equilibriu $E^{*}$ of system of Equations (1)-(3) is asymptotically stable for all $\tau \geq 0$.

(ii) If either $r<0$ or $r \geq 0$ and $\Delta>0, x_{1} *>0$ and $h\left(x_{1}^{*}\right) \leq 0$ holds then the equilibrium $E^{*}$ of system of Equations (1)-(3) is asymptotically stable for all $\tau \varepsilon\left[0 ; \tau_{0}\right)$.

(iii) If all conditions as stated in (ii) and $h^{\prime}\left(x_{0}\right) \neq 0$ hold, then system of Equation (1)-(3) undergoes a Hopf bifurcation at the equilibrium $E^{*}$, when $\tau=\tau_{j}, j=$ $0,1,2, \ldots \ldots$

\section{Numerical Results}

In this section we used MATLAB to perform some numerical simulation on system with delay and without delay. Firstly in the absence of time delay, we took some parameter values as

$r_{1}=2.5 ; r_{2}=2.55, k=20, \alpha_{1}=0.01 ; \rho_{1}=0.66 ; \alpha_{2}=0.02 ; \rho_{2}$ $=0.55 ; \gamma_{1}=0.43 ; \gamma_{2}=0.21 ; d=0.1 ; h_{1}=12 ; h_{2}=10.8 ; \boldsymbol{\theta}_{1}=$ $0.09 ; \boldsymbol{\theta}_{2}=0.06$.

For this set of parameter values we observed that the positive interior equilibriu m is $E^{*}(0.9127,11.0817,44.5116)$ which is asymptotically stable (see Fig. 1).
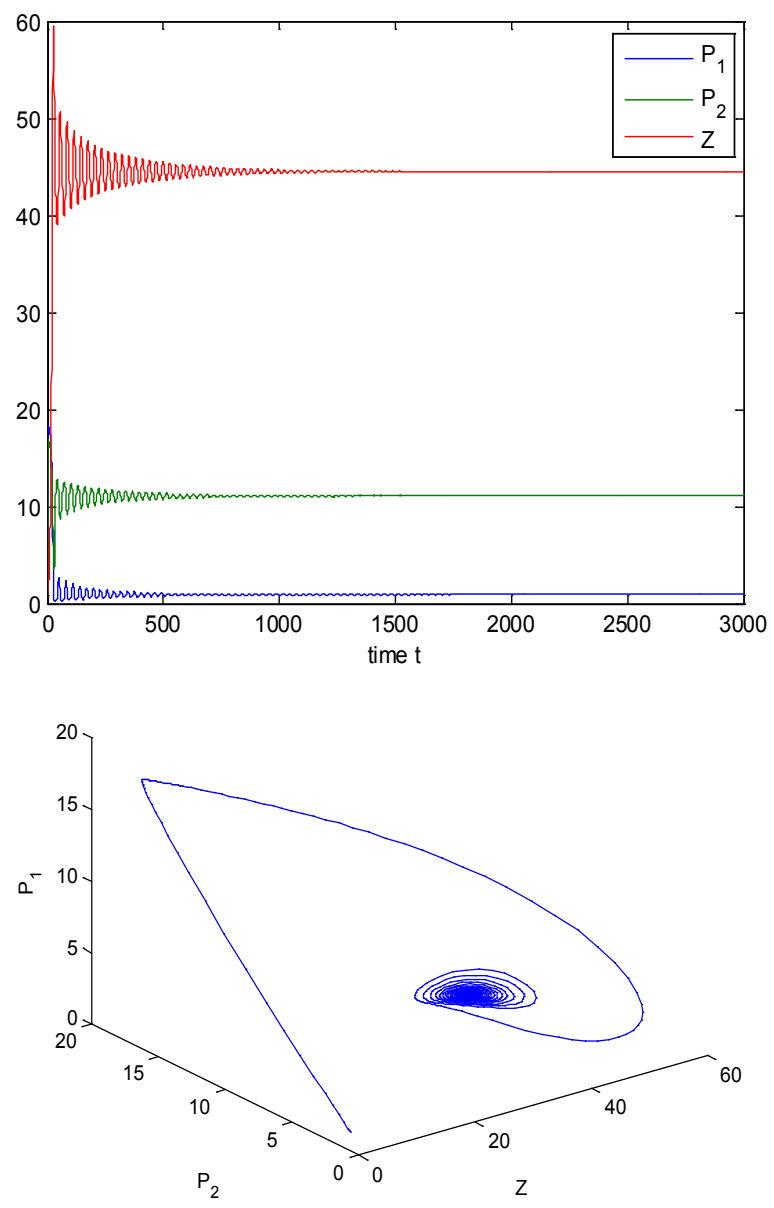

Figure 1. The positive interior equilibrium point $E^{*}(0.9127,11.0817$, 44.5116) of system without delay is asymptotically stable

We studied the Hopf bifurcation of the system of Equations (4)-(6) taking $\rho_{2}$ as the bifurcation parameter, the transversality condition hold with these parameter when $\rho_{2}=\rho_{2} *=0: 53106$. Further, fro $m$ the above process, we can determine the stability and direction of periodic solutions bifurcating from the positive equilibrium at the critical point $\rho_{2}{ }^{*}$. For instance, when

$$
\begin{gathered}
\rho_{2}=\rho_{2} *=0.53106, C_{1}(0)=-0.0000269059-0.000139517 i, \\
\operatorname{Re}\left\{\lambda\left(\rho_{2} *\right)\right\}=-0.0841233 .
\end{gathered}
$$

It follows from (14) that $\mu_{2}<0$ and $\beta_{2}<0$. Therefore, the bifurcation takes place when $\rho_{2}$ crosses $\rho_{2} *$ to the left $\left(\rho_{2}<\rho_{2}{ }^{*}\right)$, and the corresponding periodic orbits are orb itally asymptotically stable, as depicted in Fig. 2 and Fig. 3. 

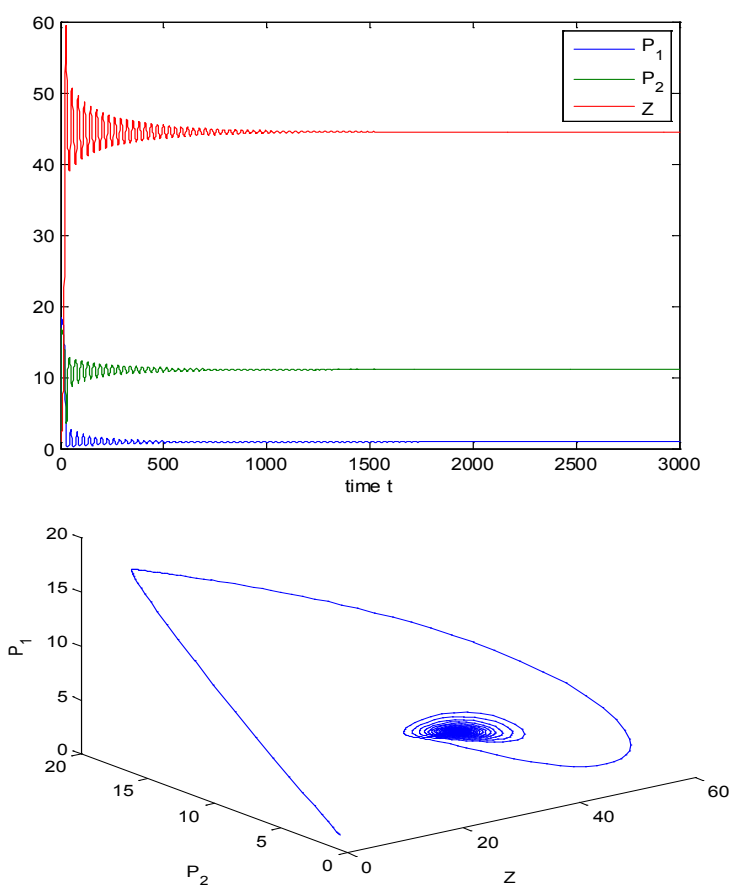

Figure 2. The positive interior equilibrium point $E^{*}$ of system without delay is asymptotically stable when $\rho_{2}=0.55>\rho_{2}{ }^{*}=0.531$
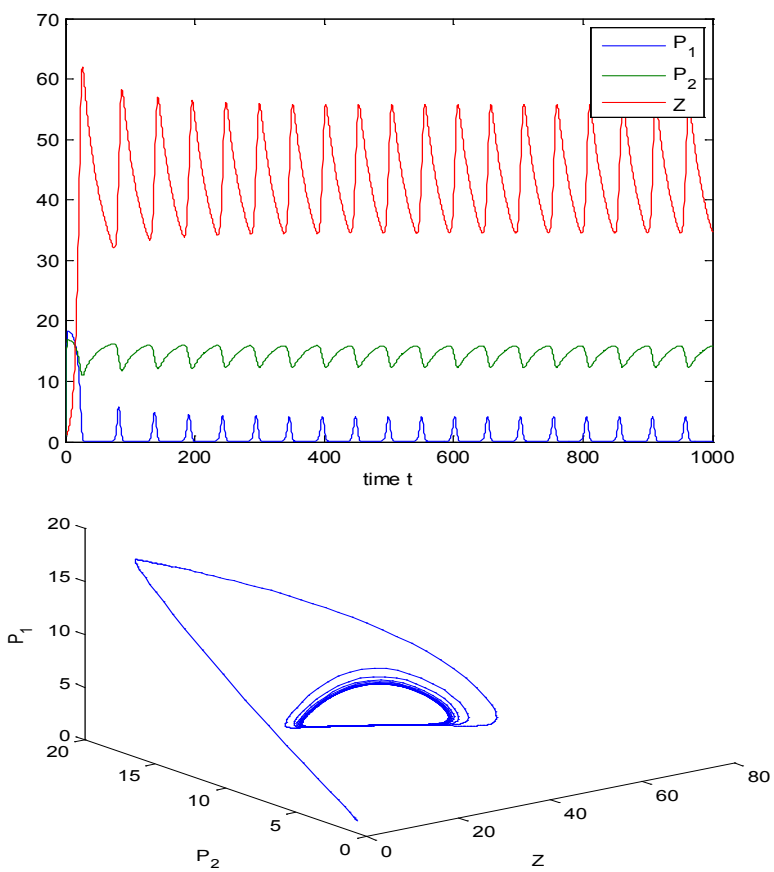

Figure 3. When $\rho_{2}=0.4<\rho_{2}^{*}=0.531$, the positive interior equilibrium point $E^{*}$ of system without delay loses its stability and a Hopf-bifurcation occurs. Further, the bifurcat ing periodic solution is orbitally, asymptotically stable.

Further now take the value of $\rho_{2}=0.55>\rho_{2} *=0.53106$ then system of Equations (4)-(6) is stable, so we have studied the Hopf-bifurcation of the system of Equations (4)-(6) taking $\rho_{1}$ as the bifurcation parameter, the transversality condition hold with these parameter when $\rho_{1}=\rho_{1} *$
$=0.683535$. Further, from the above process, we can determine the stability and direction of periodic solutions bifurcating fro $m$ the positive equilibriu $m$ at the critical point $\rho_{1}^{*}$. For instance, when

$\rho_{1}=\rho_{1} *=0.683535, C_{1}(0)=-0.0000123894-0.000006 .6961 i$, $\operatorname{Re}\left\{\lambda^{1}\left(\rho_{1} *\right)\right\}=0.11223$.
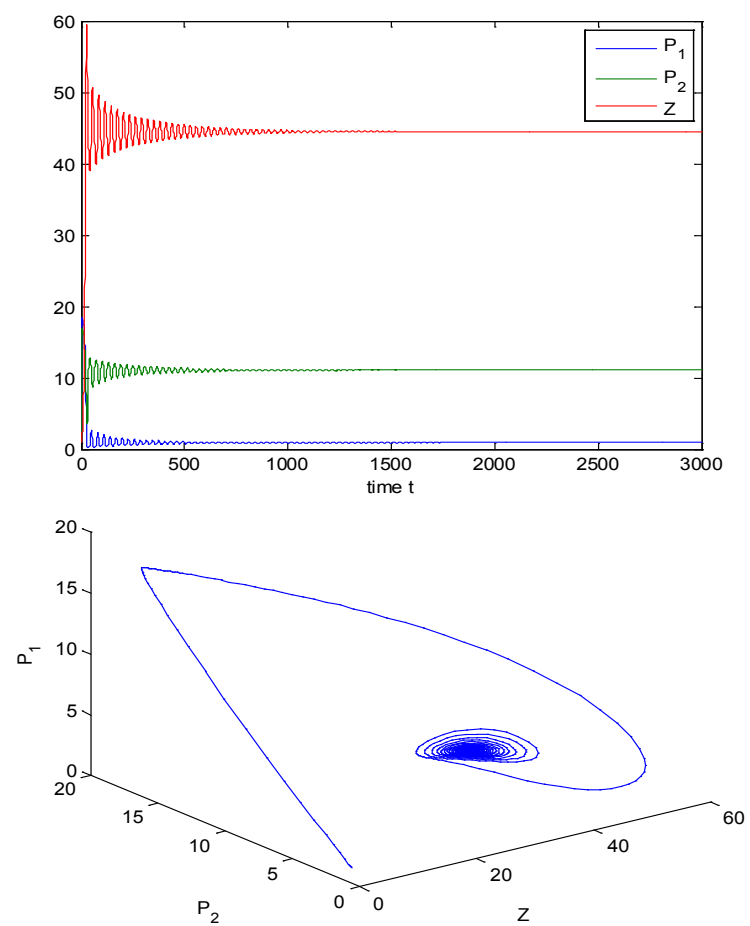

Figure 4. The positive interior equilibrium point $E^{*}$ of system without delay is asymptotically stable when $\rho_{1}=0.66<\rho_{1}^{*}=0.683535$
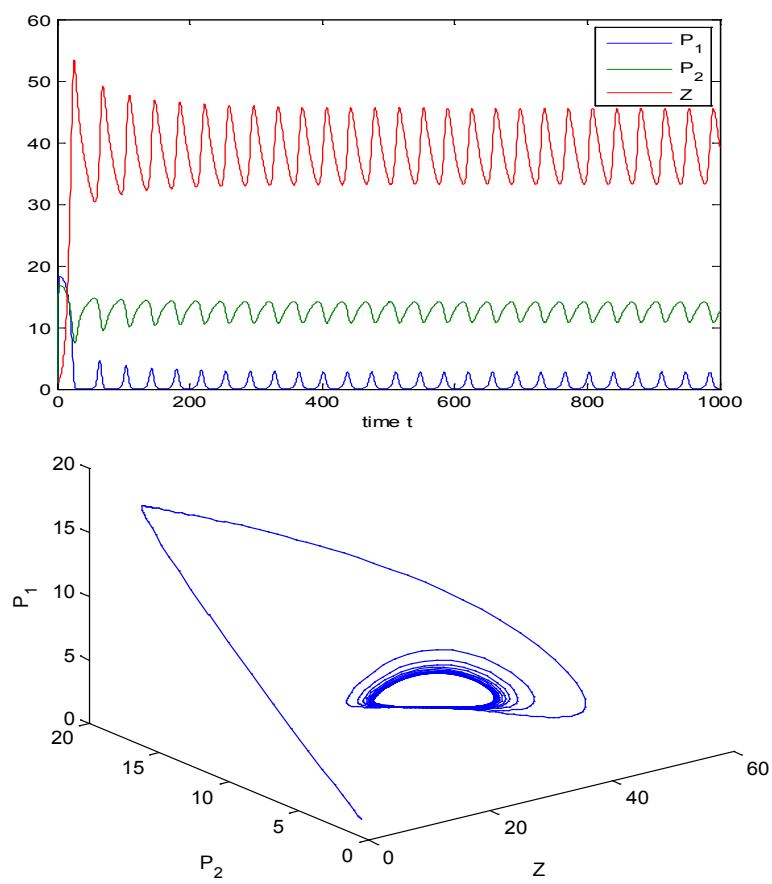

Figure 5. When $\rho_{1}=0.75>\rho_{1}^{*}=0.683535$, the positive interior equilibrium point $E *$ of system (1) loses its stability and a Hopf-bifurcation occurs. Further, the bifurcating periodic solution is orbitally, asymptotically stable 
It follows from (18) that $\mu_{2}>0$ and $\beta_{2}<0$. Therefore, the bifurcation takes place when $\rho_{1} \operatorname{crosses} \rho_{1} *$ to the right $\left(\rho_{1}>\right.$ $\left.\rho_{1}^{*}\right)$, and the corresponding periodic orbits are orbitally asymptotically stable, as depicted in Fig. 4 and Fig. 5.

If we take $\rho_{1}=0.66<\rho_{1} *=0.683535$ and $\rho_{2}=0.55>\rho_{2} *=$ 0.53106 and taking $h_{1}$ as the bifurcation parameter, the transversality condition hold with these parameter when $h_{1}=$ $h_{1}^{*}=11: 7554$. Further, from the above process, we can determine the stability and direction of periodic solutions bifurcating fro $m$ the positive equilibriu $m$ at the critical point $h_{1}^{*}$. For instance, when

$$
\begin{gathered}
h 1=h_{1}^{*}=11.7554, C_{1}(0)=-0.00002467-0.0001229 i, \\
\operatorname{Re}\left\{\lambda\left(h_{1}^{*}\right)\right\}=-0.0124335 .
\end{gathered}
$$

It follows from (22) that $\mu_{2}<0$ and $\beta_{2}<0$. There fore, the bifurcation takes place when $h_{1}$ crosses $h_{1}^{*}$ to the left $\left(h_{1}<\right.$ $h_{1}^{*}$ ), and the corresponding periodic orbits are orbitally asymptotically stable, as depicted in Fig. 6 and Fig. 7.

If we take $\rho_{1}=0.66<\rho_{1}^{*}=0.683535, \quad \rho_{2}=0.55>$ $\rho_{2}{ }^{*}=0.53106$ and $h_{1}=12>h_{1}^{*}=11.7554$, taking $h_{2}$ as the bifurcation parameter, the transversality condition hold with these parameter when $h_{2}=h_{2}^{*}=11.2643$. Further, from the above process, we can determine the stability and direction of periodic solutions bifurcating from the positive equilibrium at the critical point $h_{2}^{*}$. For instance, when $h_{2}=$ $h_{2}^{*}=11: 2643, C_{1}(0)=-0.0000283042-0.000129115 i, \operatorname{Re}\{\lambda$ $\left.\left(h_{2}^{*}\right)\right\}==0.00253856$. It follows from (24) that $\mu_{2}>0$ and $\beta_{2}$ $<0$. Therefore, the bifurcation takes place when $h_{2}$ crosses $h_{2}^{*}$ to the right $\left(h_{2}>h_{2}^{*}\right)$, and the corresponding periodic orbits are orb itally asymptotically stable, as depicted in Fig. 8 and Fig. 9.
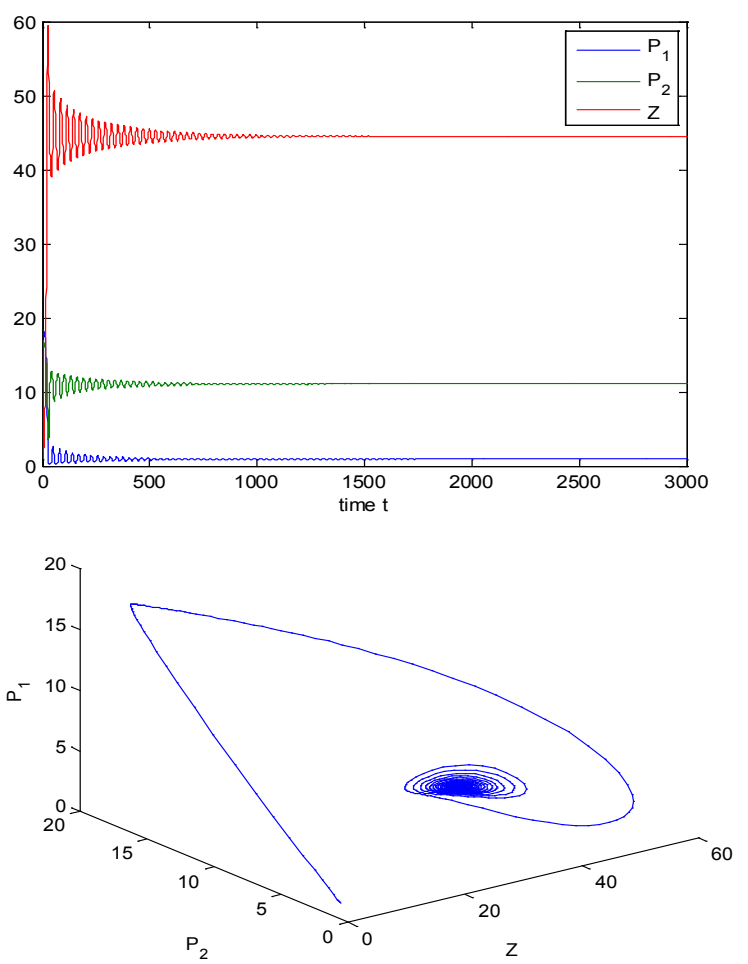

Figure 6. The positive interior equilibrium point $E^{*}$ of system (1) is asymptotically stable when $h_{1}=12>h_{1}{ }^{*}=11: 7554$
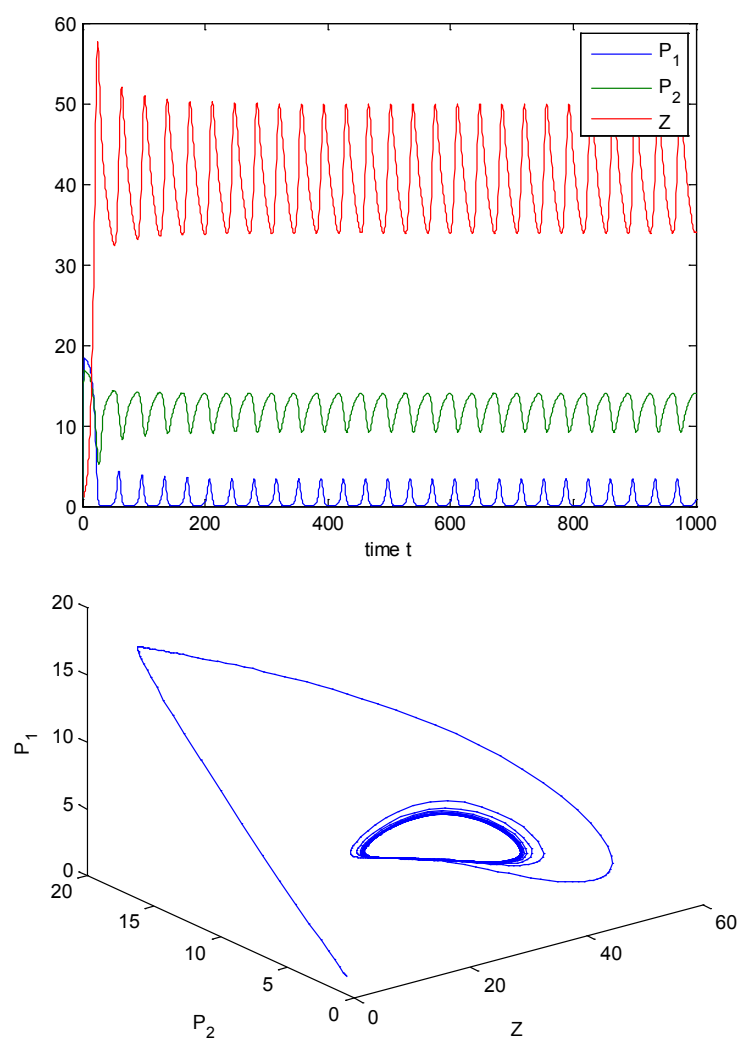

Figure 7. When $h_{1}=11<h_{1}{ }^{*}=11.7554$, the positive interior equilibrium point $E^{*}$ of system (1) loses its stability and a Hopf-bifurcation occurs. Further, the bifurcat ing periodic solution is orbitally, asymptotically stable
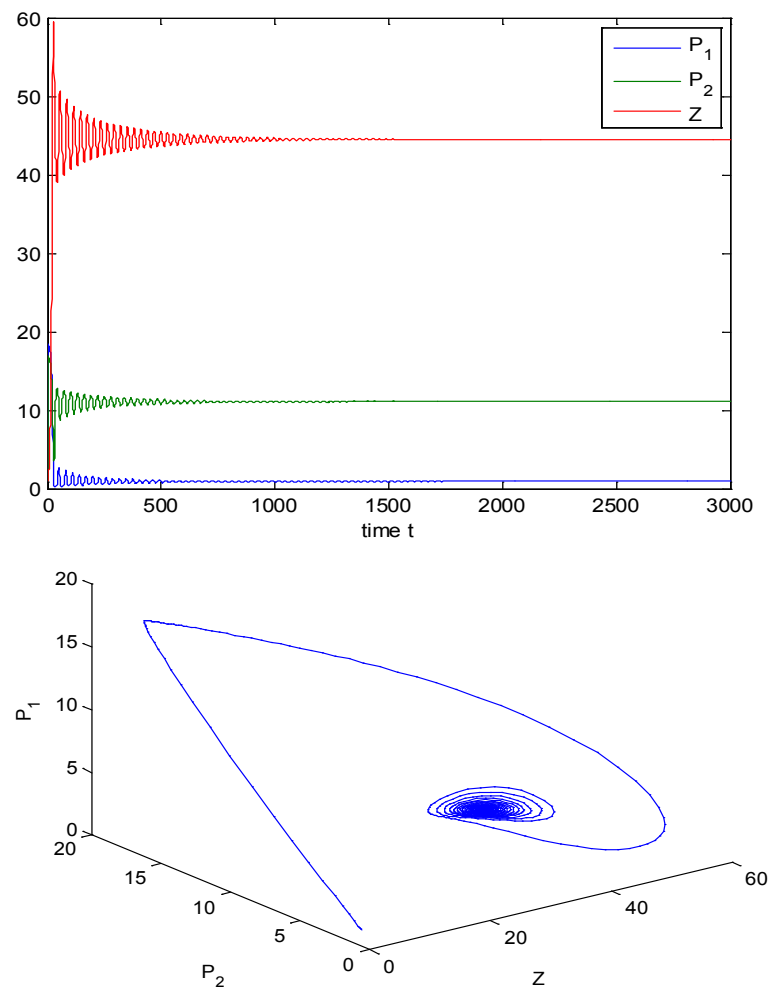

Figure 8. The positive interior equilibrium point $E^{*}$ of system (1) is asymptotically stable when $h_{2}=10: 8>h_{2}{ }^{*}=11.2643$ 

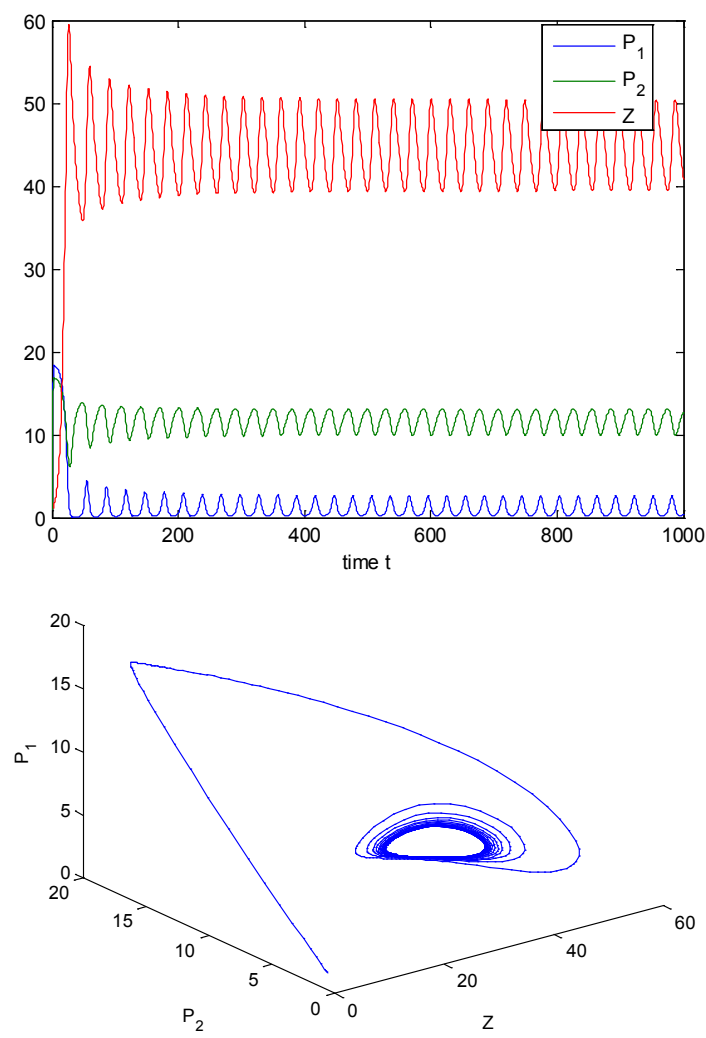

Figure 9. When $h_{2}=12>h_{2}{ }^{*}=11.2643$, the positive interior equilibrium point $E^{*}$ of system (1) loses its stability and a Hopf-bifurcation occurs. Further, the bifurcat ing periodic solution is orbitally, asymptotically stable
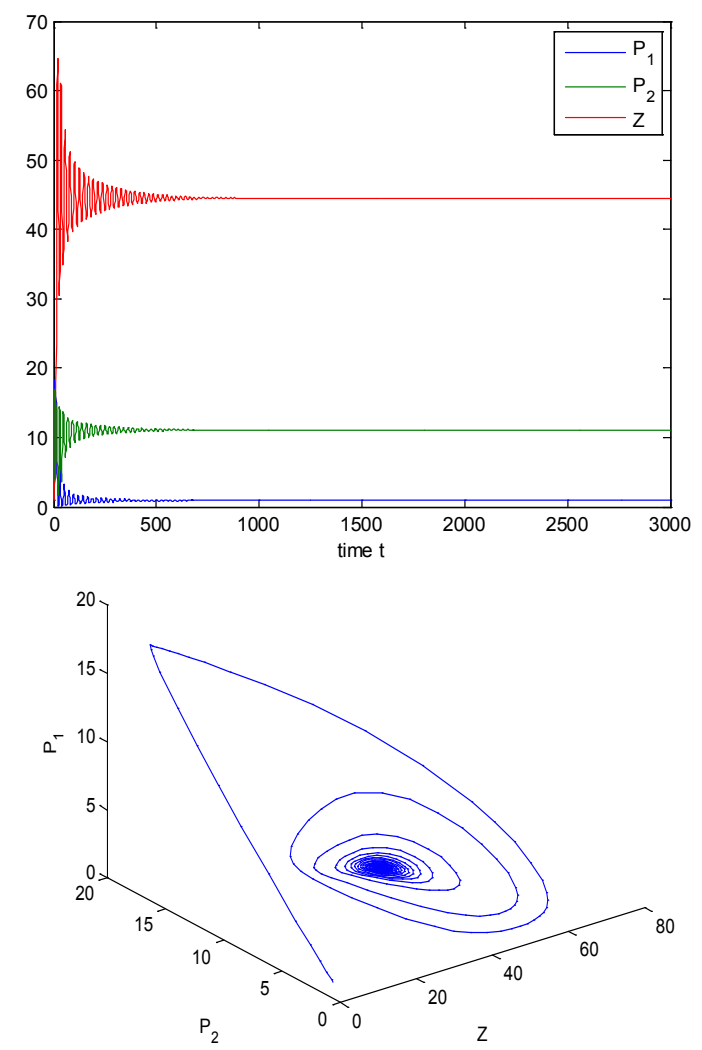

Figure 10. The positive interior equilibrium point $E *$ of system (1) is asymptotically stable when $\tau=9<\tau_{0}=9.40825$
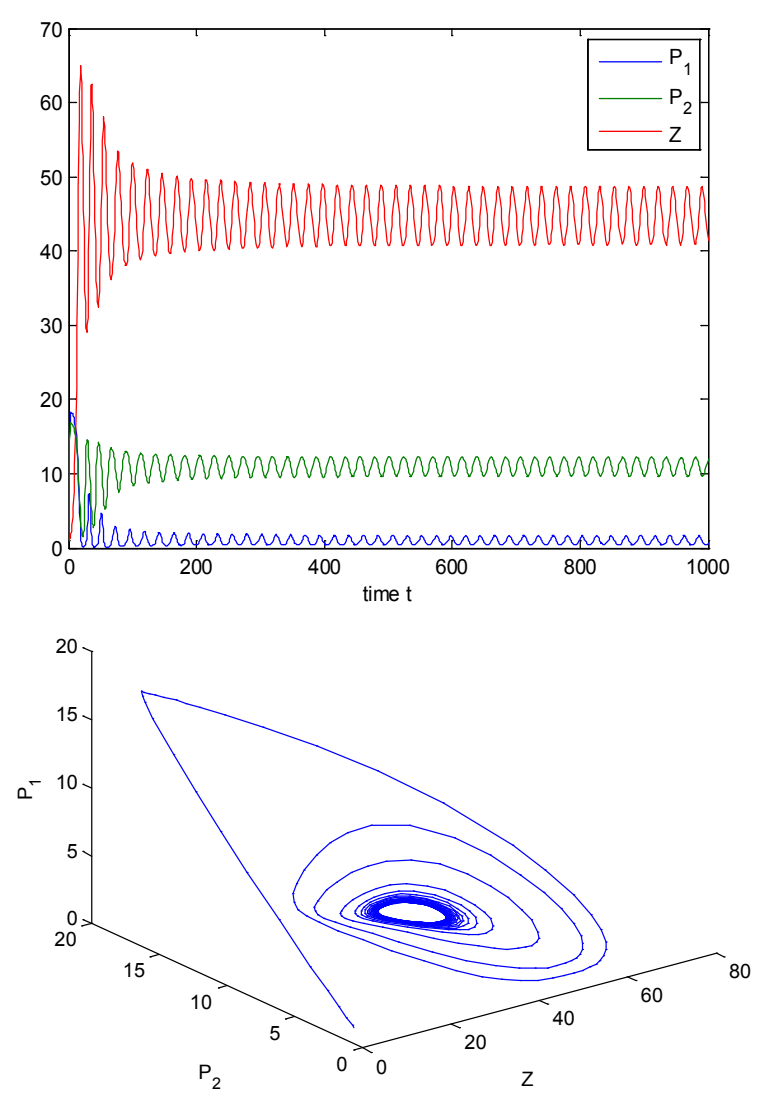

Figure 11. When $\tau=9.8>\tau_{0}=9.40825$, the positive interior equilibrium point $E^{*}$ of system (1) loses its stability and a Hopf-bifurcation occurs

If we take $\rho_{1}=0.66<\rho_{1} *=0.683535, \rho_{2}=0.55>\rho_{2}^{*}=$ $0.53106, h_{1}=12>h_{1}^{*}=11.7554$, and $h_{2}=10.8<h_{2}^{*}=$ 11.2643 the interior equilibrium point of the system becomes stable then we will study the role of time lag in the system. So we take delay as a bifurcation parameter and find out the critical value of de lay parameter $\tau=\tau_{0}=9.40825$, the interior equilibrium point loses its its stability and Hopf-bifurcation occurs, as depicted in Fig. 10 and Fig. 11.

\section{Discussion}

In the previous studies, researchers have established with the help of experimental results and mathematical modeling that toxin-producing phytoplankton may be used as a controlling agent for the termination of planktonic bloom. But those studies do not contain the presence of two harmful phytoplankton in such situation. Moreover, the effect of time lags due to liberation of toxic substances and role of Holling-type II functional response cannot be ignored in this context. In this paper we have proposed and analyzed a three component model consisting of two competing harmful phytoplankton and a zooplankton. We have studied the stability behavior of the system around the feasible steady states. Our theoretical as well as numerical results show that for a certain threshold of the system parameters, the system 
possesses asymptotic stability around the positive interior equilibriu m depicting the co-exis tence of all the three species. From qualitative and numerical analys is we find that $\rho_{1}$ and $\rho_{2}$ are bifurcating parameters for which the interior equilibrium point shows stable bifurcating solution when $\rho_{1}$ is greater than the threshold value $\rho_{1}^{*}$ and $\rho_{2}$ is less than the threshold value $\rho_{2}^{*}$ respectively. Similarly, we also find that $h_{1}$ and $h_{2}$ are bifurcating parameters for which the interior equilibrium point shows stable bifurcating solution when $h_{1}$ is less than the threshold value $h_{1}^{*}$ and $h_{2}$ is greater than the threshold value $h_{2}^{*}$ respectively. However, it is interesting to note that the dynamical nature of the bifurcating solution depends upon the parameters $\rho_{1}, \rho_{2}, h_{1}$ and $h_{2}$. When the time-lag is considered in the system, it is observed that the stable interior equilibrium point again exhibits Hopf-bifurcation for certain critical value of delay parameter.

\section{ACKNOWLEDGEMENTS}

Appreciation is extended toward the C. S. I. R., New Delhi, India, for their financial support, without which this res earch effort would not have been possible.

\section{REFERENCES}

[1] D. Anderson, "Toxic algal blooms and red tides: a global perspective In: Red Tides: Biology”, Environ. Sci. Technol. New York 11-16, 1989.

[2] G. Hallegraeff, " A review of harmful algae blooms and the apparent global increase”, Phycologia, 32, 79-99, 1993.

[3] T. Smayda, Novel and nuisance phytoplankton blooms in the sea: evidence for a global epidemic. Toxic marine phytoplankton. New York, Elsevier,1990.

[4] J. Blaxter, A. Southward, Advances in marine biology, Academic Press, London 1997.

[5] D. Huang, H. Wang, J. Feng, Z. Zhu, "Hopf bifurcation of the stochastic model on HAB nonlinear stochastic dynamics", Chaos, Solitons and Fractals 27 (4), 1072-1079, 2006.

[6] R.R. Sarkar, B. Mukhopadhyay, R. Bhattacharyya, S. Banerjee, "Time lags can control algal bloom in two harmful phytoplankton-zooplankton system", Appl. Math. and Comput. 186 (1), 445-459, 2007.

[7] J. Zhao, J. Wei, "Stability and bifurcation in a two harmful phytoplankton- zooplankton system", Chaos, Solitons and Fractals 39 (3), 1395-1409, 2009.

[8] Z. Li, F. Chen, "Extinction in periodic competitive stage-structured Lotka-Volterra model with the effects of toxic substances", J. Comput. Appl. Math. 231 (1), 143-153, 2009 .

[9] Z. Liu, L. Chen, “ Positive periodic solution of a general discrete non-autonomous difference system of plankton allelopathy with delays", J. Comput. Appl. Math. 197 (2), 446-456, 2006.

[10] J. Chattopadhyay, R.R. Sarkar, S. Mandal, “ Toxin-producing plankton may act as a biological control for planktonic blooms-field study and mathematical modeling", . J. Theor. Biol. 215, 333-44, 2002.

[11] S. Khare, O.P. Misra, J. Dhar, " Role of toxin producing phytoplankton on a plankton ecosystem", Nonlinear Analysis: Hybrid Systems 4 (3), 496-502, 2010.

[12] K. Keating, "Algal metabolite influence on bloom sequence in eutrophic freshwater ponds", Ecological Monograph Series, EPA. 600/3-76-081, Govt. Print. OH, Washington, DC, 148-152, 1976.

[13] [K. Kirk, J. Gilbert, " Variation in herbivore response to chemical defenses: zooplankton foraging on toxic cy anobacteria", Ecology 73, 2208-2217, 1992.

[14] E. Odum, Fundamentals of Ecology, W.B.Saunders Company, Philadelphia, 1971.

[15] A. Boney, Phytoplankton, Edward Arnold Ltd.,London, 1976.

[16] R.R. Sarkar, H. Malchow, "Nutrients and toxin producing phytoplankton control algal blooms a spatiotemporal study in a noisy environment", Journal of Biosciences 30 (5) 749-760, 2005.

[17] J. Chattopadhyay, R.R. Sarkar, A. E. Abdllaoui," A delay differential Equation model on harmful algal blooms in the presence of toxic substances", Math. Med. Biol. 19 (2) 137-61, 2002.

[18] R.R. Sarkar, S. Pal, J. Chattopadhyay, " Role of two toxin-producing plankton and their effect on phytoplankton-zooplankton system-a mathematical study supported by experimental findings", Biosystems 80 (1) 11-23, 2005.

[19] R.R. Sarkar, J. Chattopadhayay, "Occurrence of planktonic blooms under environ- mental fluctuations and its possible control mechanism-mathematical models and experimental observations", J. Theor. Biol. 224 (4), 501-516 2003.

[20] R.R. Sarkar, S. Petrovskii, M. Biswas, A. Gupta, J. Chattopadhyay," An ecological study of a marine plankton community based on the field data collected from Bay of Bengal”, Ecol. Model. 193 589-601, 2006.

[21] M. Kot, Elements of Mathematical Biology, Cambridge University Press, Cambridge, 2001.

[22] R. May, Stability and complexity in model ecosystems, Princeton University Press, 2001.

[23] P.Wangersky, W. Cunningham, "Time lag in prey-predator population models", Ecology 38 (1) 136-139, 1957.

[24] J. Murray, Mathematical biology: an introduction, Springer, 2002.

[25] B. Hassard, N. Kazarinoff, Y.Wan, Theory and applications of Hopf bifurcation, CUP Archive, 1981.

[26] J. Marsden, M. McCracken, The Hopf bifurcation and its applications, Springer-Verlag, 1976. 